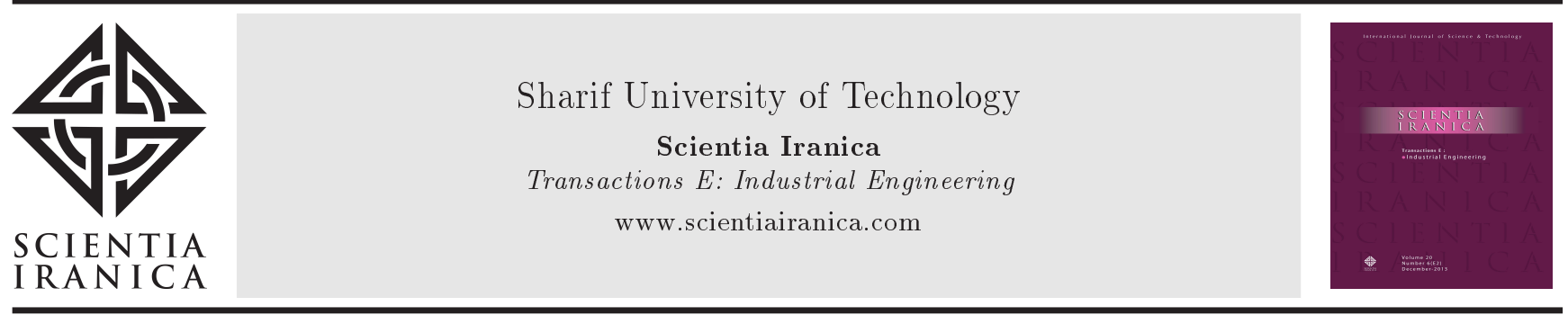

\title{
Train timetabling on double track and multiple station capacity railway with useful upper and lower bounds
}

\author{
A. Oroojlooyjadid ${ }^{\mathrm{a}, *}$ and K. Eshghi ${ }^{\mathrm{b}}$ \\ a. Department of Industrial and Systems Engineering, Harold S. Mohler Laboratory, Lehigh University, Bethlehem, PA 18015-1582, \\ USA. \\ b. Department of Industrial Engineering, Sharif University of Technology, Tehran, Iran.
}

Received 26 October 2015; received in revised form 31 August 2016; accepted 10 December 2016

\author{
KEYWORDS \\ Transportation; \\ Train timetabling; \\ Mathematical \\ programming; \\ Heuristic; \\ Lagrangian relaxation.
}

\begin{abstract}
Train scheduling has been one of the significant issues in the railway industry in recent years since it has an important role in efficacy of railway infrastructure. In this paper, the timetabling problem of a multiple-tracked railway network is discussed. More specifically, a general model is presented here in which a set of operational and safety requirements is considered. The model handles the trains overtaking in stations and considers the stations' capacity. The objective function is to minimize the total travel time. Unfortunately, the problem is NP-hard, and real-sized problems cannot be solved in an acceptable amount of time. In order to reduce the processing time, we presented some heuristic rules, which reduce the number of binary variables. These rules are based on problem's parameters, such as travel time, dwell time, and safety time of stations, and try to remove the impracticable areas of the solution space. Furthermore, a Lagrangian Relaxation algorithm model is presented in order to find a lower bound. Finally, comprehensive numerical experiments on the Tehran Metro case are reported. Results show the efficiency of the heuristic rules and also the Lagrangian Relaxation method in a way that the optimum values are obtained for all analyzed problems.
\end{abstract}

(C) 2017 Sharif University of Technology. All rights reserved.

\section{Introduction}

Railway is a fast and economic mode of transportation; according to the Association of American Railroad's study, rail companies move more than 40 percent of the US's total freight [1] and is predicted to expand the current amount by double till 2020. So, the railways managers have to expand the infrastructures or manage the current facilities more efficiently. The construction of the new infrastructures is very expensive and time-consuming, and so the utilization

*. Corresponding author. Tel.: +16109741791

E-mail addresses: afo214@lehigh.edu (A. Oroojlooyjadid); eshghi@sharif.edu (K. Eshghi)

doi: $10.24200 /$ sci. 2017.4396 efficiency of the current network facilities by optimized line planning, network timetabling, crew scheduling, and maintenance scheduling is very important. One of the most influential majors in this list is timetabling, which has engendered a big field of study by itself. In a general point of view, it can be divided into three main fields: mathematical programing, simulationbased optimization methods, and expert systems. In the field of the mathematical programing, the aim is to create the global optimal timetable or to reschedule the existing timetable. This article is focused on creating new schedule and timetables based on mathematical programming.

Higgins et al. [2] presented a mathematical model for a single-track railway with dynamic travel times. They considered delay and operational costs as the objective function and proposed a Branch and Bound 
(B\&B) to solve the problem. Kroon and Peeters [3] presented a model based on Periodic Event Scheduling Problem (PESP) in which all of their parameters, such as dwell time, headway time, and trip time, are dynamically considered. Ghoseiri et al. [4] developed a multi-objective nonlinear model to minimize fuel consumption and total passenger-time. They found the Pareto frontier and then used a distance-based method to find the solution. Zhou and Zhong [5] used the Pareto solution to solve a double-objective model and a combination of the expected waiting time and the total travel time as an objective function. A beam search in a $\mathrm{B} \& \mathrm{~B}$ algorithm is proposed to solve the MIP problem. In another study on multi-objective problems, Ping et al. [6] proposed a Particle Swarm Optimization (PSO) for dealing with the total travel time and the variation of inter departure. Vansteenwegen and Oudheusden [7] proposed an objective function consisting of different types of waiting time and the late arrival. Furthermore, they presented a two-phase algorithm, obtaining an ideal buffer time, and then a timetable is created by using an LP model. Finally, a simulation compares different timetables. Vansteenwegen and Oudheusden [8] proposed the ideal running time instead of summation of actual travel and dwell times. Their objective is based on the delay distribution of trains, the passengers count, and different types of waiting time and late arrivals. They built different timetables with a LP model and a simulation evaluates them. Li et al. [9] proposed a mixed integer model with a fuzzy multiobjective function to minimize energy minimization, carbon emission cost, and total passenger time. The model considered an improved version of objective function compared to [4]; in the situation that all trains are powered by electricity, the proposed model degenerates to the model proposed in [4].

Zhou and Zhong [10] presented a complicated $\mathrm{B} \& \mathrm{~B}$ algorithm to solve the proposed problem in [5]. They developed three methods for node selection and proposed some other complicated rules for the branching process in B\&B method. Also, they designed a Lagrangian Relaxation method and another heuristic method to find a lower bound. Lee and Chen [11] proposed a heuristic method that provides train path and timetable simultaneously on mixed single- and double-track networks. The method has four phases that iteratively creates and adjusts a timetable. For a single-line network, Castillo et al. [12] proposed a threestage method which decomposes the model to find a solution with the maximum relative time, i.e. the ratio of travel time to minimum possible travel time, the minimum sum of the departure time, and the minimum fuel consumption. In order to solve the problem, they proposed a bicriteria algorithm to minimize the relative travel time. Yang et al. [13] proposed a model to minimize the total passenger trip time, considering the number of passengers as a stochastic function. They used the expected value, pessimistic and optimistic values for the number of passengers and designed a B\&B algorithm to solve the model. Castillo et al. [14] proposed a bisection method with objective function of relative travel time and used some heuristic methods to reduce the number of inactive binary variables in single track and double track networks. Furthermore, they proved that the solution of the algorithm is optimal. Castillo et al. [15] presented a nonlinear model which considers Alternate Double Single Track (ADST) lines. Their objective is to minimize the construction cost, maximum relative travel time of all trains, and the sum of total relative travel times, such that the obtained departure times be close to the desired ones. They linearized the initial model and proposed some binary reduction.

By the graph approach, Liu and Kozan [16] modeled the train timetabling problem as a blocking parallel-machine job shop scheduling problem and introduced an improved Shifting Bottleneck Procedure. Then, they used an alternative graph to solve the main problem. Burdett and Kozan [17] introduced a graph model for parallel rails with linked rails in sidings, capacitated buffer, acceleration and deceleration times which also do not allow unforced idle time. In order to solve the problem, they used a Constructive Algorithm (CA), Simulated Annealing (SA), and Local Search (LS) metaheuristics. Burdett and Kozan [18] addressed the adjusting of timetables to handle perturbations and unnecessary multiple overtaking conflicts. They used the disjunctive graph to represent the problem and used LS and SA to obtain good correction. Burdett and Kozan [19] considered the timetabling problem as a jobshop problem and addressed a customized disjunctive graph to construct the timetable. In addition, they proposed some CA to create feasible solutions. Burdett and Kozan [20] introduced a disjunctive graph model, considering trains and sections length, headways and blocking conditions, nondelay scheduling policy, and passing loops. They proposed a CA, based on $\mathrm{NEH}$ algorithm for job shop problem, an SA, and LS methods to create and improve solutions. Caimi et al. [21] proposed a new model for solving microscopic-scale train timetabling problems. In their defined problem, stations have multiple lines and gate capacities, and the arrival and departure times of trains are known. They proposed a new method for determining large conflict cliques in conflict graph and put them in an ILP model, relaxing strongly the related LP problem. Liu and Kozan [22] proposed a disjunctive graph, considering capacitated stations and sidings on single-line networks, no wait, and blocking properties. A hybrid algorithm was proposed to construct the feasible train timetable combined by a LS algorithm, minimizing the makespan. Furini and Kidd [23] proposed a heuristic 
algorithm using relaxed dynamic programming, based on the acyclic space-time graph. The algorithm starts with an ideal timetable and tries to resolve the conflicts between trains. Their model considers single corridor networks, parallel tracks in the stations, and also the presence of junctions on the network.

In the category of meta-heuristic methods, Tormos et al. [24] proposed a Genetic Algorithm (GA) for the PESP that included a guided process to build the initial population. Jamili et al. [25] proposed a model to deal with train timetabling in single-rail networks based on PESP. They also proposed a hybrid algorithm consisting of SA and PSO. Shafia et al. [26] proposed a new robust periodic model based on PESP on a singlerail network. In their model, station capacity and headway constraints were considered. A fuzzy approach was used to consider the robustness and its trade-off with the train delay and the time interval between departures of trains from the same origin. Also, a SA was used to solve the problems. In the robust problem category, Cacchiani and Toth [27] presented a complete survey on the robust models and their features. Some new robust research studies are also presented in [28]. Yang et al. [29] proposed a mathematical programming model, considering total energy consumption and total traversing time optimization in a railway network with multiple trains and multiple links in stations. An integrated GA and simulation are used to obtain an approximate optimal strategy. Reimann and Leal [30] proposed a customized Ant Colony Optimization to minimize the total weighted tardiness in single-track railways. Xu et al. [31] proposed a Travel Advance Strategy (TAS) method combined with GA for dealing with single-track railways. The algorithm searches for a timetable with the minimal delay ratio, i.e. the total delay time over the total free-run time. Sun et al. [32] proposed a model to obtain timetabling on one-way high speed double-track networks. In order to solve the model, they proposed an improved GA and used simulation to analyze the accuracy of the algorithm. Huang et al. [33] also proposed a GA to provide the timetable of an urban rail transit system. The model adjusts the headway to obtain the best trade-off between the passenger travel time and energy consumption with a guaranteed transit capacity.

In a little bit different context, Heydar et al. [34] proposed a linear formulation of cyclic timetabling problem for single track railways in which minimization of cycle length is the objective. Barrena et al. [35] proposed a train timetabling model which deals with dynamic demand. Considering dynamic demand for different routes in different times, they proposed threebinary models and a branch and cut algorithm to minimize the total waiting of passengers in stations.

We could not find any study representing a linear mathematical model to obtain timetabling in a network with parallel unidirectional tracks and limited number of station's platforms or siding's capacities. In this way, we propose an approach that models overtaking decisions at stations/sidings, as opposed to other approaches which model precedence's (i.e., sequencing) on single tracks as limited sources. Also, we show that the problem is NP hard and the real-sized problems cannot be solved in a reasonable amount of time. In order to obtain beneficial solutions in a reasonable time, we provide some new upper bound and lower bound rules. By concentrating on these two topics, the structure of this article is as follows. In Section 2, we provide the mathematical model. Then, in Section 3, we propose the upper bound rules; in Section 4, the lower bound rule is proposed and it is followed by numerical experiment's result in a real world problem.

\section{Problem definition}

In this section, we define the problem and its assumptions, present the notations, and introduce our model.

This study considers a general situation of double line networks. In all lines and corridors in the network, there are two parallel tracks, and each track facilitates unidirectional flow and not bidirectional, i.e. there is no opposite direction train, which is a common situation in metro, subway, and high-speed networks; it is of interest as $[36,37]$ covered some earlier research studies. Each station or siding can have one or more capacity, some of which may have no platform for passengers. Also, in sidings, there is no linked rail between opposite directions and each direction has its separate sidings. In addition, overtaking is allowed and trains can only overtake each other in the siding (passing loops) or in the stations with more than one platform, i.e. train routes in stations are not fixed. Moreover, the main line is the line that connects the stations or siding with each other. A general view of the siding and main line is showed in Figure 1.

There may be different types of trains with different speeds and dwell times. The trains may be freight- or passenger specific or any mixture of them. It is assumed that for each train in each station of the network, there is minimum and maximum dwell times, and also travel time between two consecutive stations or sidings is known. Routes and the dispatching sequence of trains between stations and sidings are

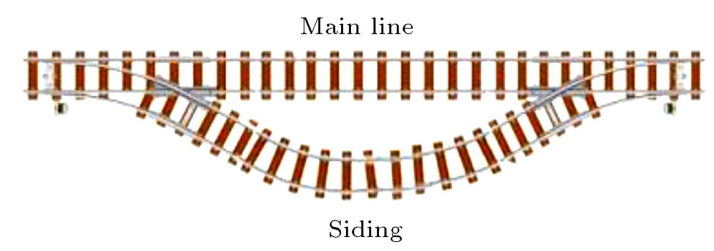

Figure 1. Main line and siding. 
given. In addition, the travel time between two stations or sidings is the difference between the departure times of the last station until the arrival time of the current station. Considering the type of trains and their intrinsic nature in metro and high-speed networks, there is no significant time for acceleration and deceleration times, but we have considered the minimum travel time between two stations to include these times. Note that the considered parameters, e.g. travel time, dispatching sequence, and dwelling time, are known in any railway network, and they are the most common features of the MIP-models for the timetabling problem.

A mathematical model is presented as follows. In this model, output is the timetable of a railway network. The most important assumptions of our model are as follows:

- All parameters of the model are deterministic;

- Each corridor of the network has one line in each direction;

- The station capacity, which is the number of platforms in the stations, can be any positive integer number. The same situation exists for sidings;

- Acceleration and deceleration times are considered in the travel time;

- Number of trains and their routes is given.

\subsection{Notation}

Throughout the paper, we reserve $l$ to denote line index in a network, $L$ is the list of all lines, $t$ defines train index, $T_{l}$ is the list of trains in line $l, s$ is the station index in each line, and $S_{l}$ defines the total number of station(s) in line $l$. Also, $q$ is the next station in line $l$, i.e. $q=s+1$. Finally, $m_{l}$ denotes the last station in line $l$. The major parameters of our problem are as follows:

$S F_{l, s} \quad$ Minimum headway (safety time) between two adjacent trains in station $s$ at line $l$

$S C_{s} \quad$ Capacity of station $s$, which is the number of platform tracks and capacity of stops

$\underline{D}_{l, s}^{t} \quad$ Minimum dwell time of train $t$ in station $s$ of line $l$

$\bar{D}_{l, s}^{t} \quad$ Maximum dwell time of train $t$ in station $s$ of line $l$

$\underline{T}_{l, s, q}^{t} \quad$ Minimum travel time of train $t$ between stations $s$ and $q(s+1)$ of line $l$

$\bar{T}_{l, s, q}^{t} \quad$ Maximum travel time of train $t$ between stations $s$ and $q(s+1)$ of line $l$

$r_{l, t, s} \quad$ Earliest start time. Minimum start time of train $t$ in station $s$ of line $l$.

$M \quad$ A large positive number
Also, we use these two notations throughout the paper:

$\Psi_{t, t^{\prime}, q} \quad$ The time interval between the departure time of train $t$ from station $q$ and the arrival time of train $t^{\prime}$ to this station

$\vartheta_{t, t^{\prime}, s} \quad$ The time interval between the departure time of two trains from station $s$

For safety reasons, trains are not permitted to get close to each other in case of collision. Between departure and arrival of trains in each station or siding, minimum headway must be satisfied. Other situations are: departure of two trains from a station or siding and arrival of two trains in a station or siding. Stations' headway is a predefined parameter that is inherently related to geographical and technical characteristics of each station. In order to identify the decision variables, first, we define the situations where every two trains may encounter in a station or siding. These situations are the events that may bring some changes to the sequence of any two arbitrary trains. With regard to the situations of the problem, for each two trains, four different situations can occur. Event 1 defines the situation that train $t$ overtakes train $t^{\prime}$ in station $s$. In Event 2, train $t^{\prime}$ overtakes train $t$ in station $s$. In Event 3 , train $t^{\prime}$ arrives in and departs from station $s$ after train $t$. Finally, Event 4 demonstrates the situation that train $t^{\prime}$ arrives in and departs from station $s$ before train $t$. In other words, in this event, train $t$ has overtaken train $t^{\prime}$ before station $s$ or is scheduled before train $t^{\prime}$ from the first station. Figure 2 shows and clarifies the mentioned situations for trains $t$ and $t^{\prime}$.

By considering these events and situations, the decision variables are as follows, covering any situation in a network:

$\begin{array}{ll}c_{l, t, s} & \begin{array}{l}\text { Departure time of the } t \text { th train from } \\ \text { station } s \text { of line } l\end{array} \\ s_{l, t, s} & \text { Arrival time of the } t \text { th train in station } \\ & s \text { of line } l \\ x_{t, t^{\prime}, s} & 1 \text { if train } t^{\prime} \text { overtakes train } t \text { in station } \\ & s \text { as Events } 1 \text { and } 2 \text {, otherwise } 0 \\ x_{t, t^{\prime}, s}^{\prime} & 1 \text { if train } t^{\prime} \text { is scheduled after train } t \text { in } \\ & \text { station } s, \text { otherwise } 0 \text {. This variable } \\ & \text { represents the situations of Events } 3 \\ & \text { and } 4 . \\ & 1 \text { if trains } t^{\prime} \text { and } t \text { use station } s \\ y_{t, t^{\prime}, s} & \text { simultaneously, otherwise } 0 .\end{array}$

As the definition of decision variables shows, binary variables will model overtaking decisions in a double line unidirectional railway network. By this approach, our decision variables define the sequence of trains in the network and determine when and which 


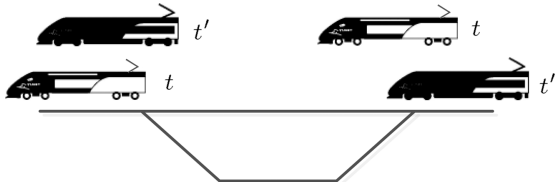

(a)

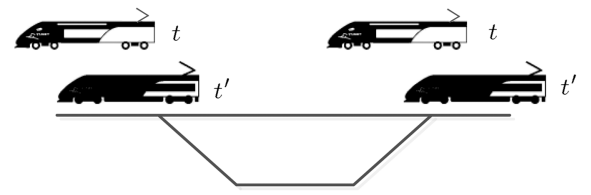

(c)

Figure 2. The situation of two trains in each event type:

station or siding the trains will overtake each other. Somehow, this is a different approach compared to the current models that define precedence and sequencing on single tracks. With regard to these variables, constraints of the problem are defined as follows:

\subsection{Objective function}

Our model's aim is to minimize the summation of the departure time in the last station for all trains, which is the minimum total travel time as is shown in Eq. (1).

$$
\min z=\sum_{l=1}^{L} \sum_{t=1}^{T} c_{l t m_{l}} .
$$

This objective function tries to increase the efficacy of the network's infrastructure and also minimize the travel time. Therefore, it satisfies the criteria of two main groups in the railway networks, i.e. passengers and railway owners. It is also a commonly used objective function in research studies of $[3,5,10,12,36,38,39]$.

\subsection{Travel time constraints}

The constraints below ensure that the running time of a train does not violate a given limit of time, which also defines the speed limitation on the line. Furthermore, they do not allow the running time to drop below a specific amount of time defined by the desire of passengers. For all $t \in T_{l}, \forall l \in L$, and $\forall s \in S_{l}$, the constraints are as follows:

$$
\begin{aligned}
& s_{l, t, s+1} \geq c_{l t s}+\underline{T}_{l, s, q}^{t}, \\
& s_{l, t, s+1} \leq c_{l t s}+\bar{T}_{l, s, q}^{t} .
\end{aligned}
$$

\subsection{Dwell time constraints}

The following constraints ensure that the dwell time of a train does not violate a given limit of time. The upper and lower bounds must be gathered as real values for each station and train. Moreover, for trains that have not been scheduled to stop at a given station, both bounds are set to zero for that station. For all $t \in T_{l}, \forall l \in L$, and $\forall s \in S_{l}$, the constraints are as follows:

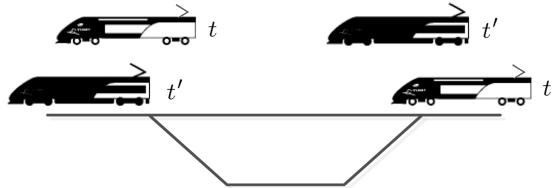

(b)

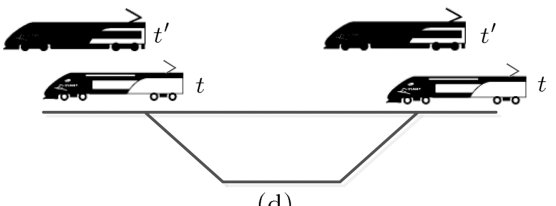

(d)

(a) Event 1, (b) Event 2, (c) Event 3, and (d) Event 4.

$$
\begin{aligned}
& c_{l t s} \geq s_{l t s}+\underline{D}_{l, s}^{t}, \\
& c_{l t s} \leq s_{l t s}+\bar{D}_{l, s}^{t} .
\end{aligned}
$$

\subsection{Overtaking constraints}

The constraints below aim to define the sequence of trains in each corridor and station and also to determine the arrival and departure times of trains. Using the defined binary variables, the related constraints of each event have been defined. In Events 3 and 4, Constraints (6) and (7) ensure that the arrival and departure times of train $t^{\prime}$ be greater than train $t$ if train $t^{\prime}$ is scheduled after train $t$, and vice versa. Also, in this situation, Constraint (8) does not allow trains to overtake each other except in the stations. Constraints (9) and (10) ensure that the arrival time of train $t^{\prime}$ be greater than that of train $t$ and its departure be lower than that of train $t$ if train $t^{\prime}$ is scheduled for the overtaking of train $t$. They act the same as description of Events 1 and 2. Constraint (11) acts similarly to Constraint (8) and does not allow, in Events 1 and 2 trains, to overtake each other except in the stations. Constraint (12) ensures that in each station and for each pair of trains $t$ and $t^{\prime}$, exactly one even $t$ can occur. In order to simplify the notation, we used $\Phi_{l, s}\left(t, t^{\prime}\right)$ to represent $S F_{l, s}-\left(1-x_{t, t^{\prime}, s}^{\prime}\right) M$. For all $l \in L, \forall t, t^{\prime} \in T_{l}$, and $\forall s \in S_{l}$, the constraints are as follows:

$$
\begin{aligned}
& s_{l t^{\prime} s} \geq s_{l t s}+\phi_{l, s}^{\prime}\left(t, t^{\prime}\right) ; \quad \forall t \neq t^{\prime}, \\
& c_{l t^{\prime} s} \geq c_{l t s}+\phi_{l, s}^{\prime}\left(t, t^{\prime}\right) ; \quad \forall t \neq t^{\prime}, \\
& s_{l t^{\prime} s} \geq s_{l t s}+\phi_{l, s}^{\prime}\left(t, t^{\prime}\right) ; \quad s>1 ; \forall t \neq t^{\prime}, \\
& s_{l t^{\prime} s} \geq s_{l t s}+\phi_{l, s}\left(t, t^{\prime}\right) ; \quad \forall t \neq t^{\prime}, \\
& c_{l t s} \geq c_{l t^{\prime} s}+\phi_{l, s}\left(t, t^{\prime}\right) ; \quad \forall t \neq t^{\prime}, \\
& s_{l t s} \geq s_{l t^{\prime} s}+\phi_{l, s}\left(t, t^{\prime}\right) ; \quad s>1 ; \forall t \neq t^{\prime}, \\
& x_{t^{\prime}, t, s}^{\prime}+x_{t, t^{\prime}, s}^{\prime}+x_{t, t^{\prime}, s}+x_{t^{\prime}, t, s}=1 ; \forall t \neq t^{\prime} .
\end{aligned}
$$




\subsection{Station capacity constraints}

This set of constraints is proposed to guarantee the maximum capacity constraint of each station or siding. Constraint (13) assures that in a station with one capacity, only one train stops in station at any time (Events 3 and 4). Thus, the arrival time of one train must be greater than that of another one which results in the condition that more than one train cannot be stopped at one moment in the station:

$$
\begin{gathered}
s_{l t s} \geq c_{l t^{\prime} s}+\phi_{l, s}^{\prime}\left(t^{\prime}, t\right) \\
s_{l t^{\prime} s} \geq c_{l t s}+\phi_{l, s}^{\prime}\left(t, t^{\prime}\right) ; \quad S C_{s}=1 ; \quad \forall t>t^{\prime}, \\
\left(x_{t_{1}, t_{2}, s}+x_{t_{2}, t_{1}, s}\right)+\left(x_{t_{1}, t_{3}, s}+x_{t_{3}, t_{1}, s}\right)+\ldots \\
+\left(x_{t_{1}, t_{S C_{s}+1}, s}+x_{t_{S C_{s}+1}, t_{1}, s}\right)+\ldots \\
+\left(x_{\left.t_{S C_{s}, t_{S C_{s}+1}, s}+x_{t_{S C_{s}+1}, t_{S C_{s}, s}}\right)}\right. \\
\leq\left(\begin{array}{c}
\left(S C_{s}+1\right) \\
2
\end{array}\right) \\
-1 ;\left\{\begin{array}{l}
\forall l \in L \\
\forall t_{1}, t_{2} \ldots t_{S C_{s}+1} \in T_{l} \\
\forall s \in S_{l} \quad \text { and } \quad S C_{s}>1
\end{array}\right.
\end{gathered}
$$

Constraint (14) considers the capacity constraint of station $s$ with capacity of $S C_{s}$ for the overtaking of events (Events 1 and 2). In stations whose capacity is one, Constraint (13) does not allow for any overtaking, i.e. the constraint is $\left(x_{t, t^{\prime}, s}+x_{t^{\prime}, t, s}\right) \leq 0$ for all of $t \neq$ $t^{\prime} \in T_{l}$ and $\forall s \in S_{l}$ in line $l$. In stations with one more capacity, the constraint considers all pair combinations of three trains (because $\left(\begin{array}{c}S C_{s}+1 \\ 2\end{array}\right)=3$ ) as:

$$
\begin{gathered}
\left(x_{t, t^{\prime}, s}+x_{t^{\prime}, t, s}\right)+\left(x_{t, t^{\prime \prime}, s}+x_{t^{\prime \prime}, t, s}\right) \\
+\left(x_{t^{\prime}, t^{\prime \prime}, s}+x_{t^{\prime \prime}, t^{\prime}, s}\right) \leq 2,
\end{gathered}
$$

for all $t \neq t^{\prime} \neq t^{\prime \prime} \in T_{l}$ and $\forall s \quad \in S_{l}$ in line $l$. This constraint ensures that at most one train can overtake another train at each time, i.e. only two trains can stop in station $s$ each time. To further illustrate the issue, consider the situation that in station $s$, which has two platforms, train $t$ is scheduled to overtake train $t^{\prime}$, i.e. $x_{t^{\prime}, t, s}=1$ and $\left(x_{t, t^{\prime}, s}+x_{t^{\prime}, t, s}\right)=1$ and train $t^{\prime \prime}$ is entering station $s$. Three situations may occur:

1. Assume that train $t^{\prime \prime}$ is scheduled to depart from station $s$ after train $t^{\prime}$. In this situation, there is no additional overtaking and train $t^{\prime \prime}$ may incur some delay;

2. Assume that without regarding the station capacity, train $t^{\prime \prime}$ is scheduled to overtake train $t$ in station $s$.
If train $t^{\prime \prime}$ overtakes train $t$, i.e. $x_{t, t^{\prime \prime}, s}=1$ and $\left(x_{t, t^{\prime \prime}, s}+x_{t^{\prime \prime}, t, s}\right)=1$ train $t^{\prime \prime}$ arrives later than trains $t$ and $t^{\prime}$ and must depart before them. In another words, train $t^{\prime \prime}$ also has to overtake train $t^{\prime}$, meaning that $x_{t^{\prime}, t^{\prime \prime}, s}=1$ and $\left(x_{t^{\prime \prime}, t^{\prime}, s}+\right.$ $\left.x_{t^{\prime}, t^{\prime \prime}, s}\right)=1$. So, $\left(x_{t, t^{\prime}, s}+x_{t^{\prime}, t, s}\right)+\left(x_{t, t^{\prime \prime}, s}+x_{t^{\prime \prime}, t, s}\right)$ $+\left(x_{t^{\prime}, t^{\prime \prime}, s}+x_{t^{\prime \prime}, t^{\prime}, s}\right)=3$, and considering the RHS of the constraint, the situation is not allowed and the assumption is not true. So, Constraint (14) does not allow for exceeding the capacity of the station;

3. Assume that train $t^{\prime \prime}$ is scheduled to overtake train $t^{\prime}$ in station $s$ and train $t$ departs from the station before arrival of train $t^{\prime \prime}$.

If trains $t^{\prime \prime}$ and $t$ overtake train $t^{\prime}$ in station $s$, i.e. $x_{t^{\prime}, t^{\prime \prime}, s}=1,\left(x_{t^{\prime \prime}, t^{\prime}, s}+x_{t^{\prime}, t^{\prime \prime}, s}\right)=1, x_{t^{\prime}, t, s}=1$, and $\left(x_{t, t^{\prime}, s}+x_{t^{\prime}, t, s}\right)=1$, the constraint is satisfied.

With a similar approach to the station with capacity of $S C_{s}$, there are $\frac{\left(S C_{s}+1\right) S C_{s}}{2}$ pairs of variables; on the right-hand side, the number of pairs minus one is replaced. This set does not allow for violation in stations' capacity with a larger capacity. Furthermore, Constraints (15) to (17) consider the station capacity when the two trains are scheduled with Event 3 or 4 :

$$
\begin{array}{r}
s_{l t^{\prime} s} \geq c_{l t s}+S F_{l, s}-\left(1-x_{t, t^{\prime}, s}^{\prime}\right) M \\
-M y_{t, t^{\prime}, s} ; \quad S C_{s} \geq 2 ; \quad \forall t>t^{\prime}, \\
\sum_{t^{\prime}, t \neq t^{\prime}}^{T}\left(y_{t, t^{\prime}, s}+y_{t^{\prime}, t, s}+x_{t, t^{\prime}, s}+x_{t^{\prime}, t, s}\right) \\
\leq S C_{s}-1 ; \quad S C_{s} \geq 2, \\
y_{t, t^{\prime}, s} \leq x^{\prime}{ }_{t, t^{\prime}, s} ; \quad S C_{s} \geq 2 ; \quad \forall t>t^{\prime} .
\end{array}
$$

As mentioned, $y_{t, t^{\prime}, s}$ is the auxiliary variable to consider the station capacity in the mentioned events. Constraints (15) and (17) ensure that the auxiliary variables can take value when Events 3 and 4 occur. For instance, in Event 3 where train $t$ is scheduled before train $t^{\prime}$, i.e. $x_{t, t^{\prime}, s}^{\prime}=1, y_{t, t^{\prime}, s}$ can take value one. Regarding Constraint (15), if $y_{t, t^{\prime}, s}$ takes $1, s_{l t^{\prime} s}$ will be greater than $M$; this means that the trains can stop simultaneously in station $s$. The same situations can be assumed for Event 4. Also, Constraint (16) ensures that the number of trains that can stop in each station will not be more than its capacity.

These constraints with continuous time variables handle the station capacity in each of the four events, and it is a new formulation in the literature. The current papers mostly consider time slotting approach, e.g. [40], which considers a time horizon for the network's operation time and discretizes it into some timestamps, and they also propose some constraints to consider the station capacity. A similar slot-based scheduling was 
used by Dessouky et al. [41] in an integrated bi-level station layout design and scheduling model.

\subsection{Departure time constraint}

It is necessary that some trains may have a predefined departure time for some stations. It may occur in the first station of the corridor. For all $l \in L, \forall t \in T_{l}$, and $\forall s \in S_{l}$, the constraint is as in Eq. (18):

$$
c_{l t s} \geq r_{l t s} \text {. }
$$

Compared to the current models of train timetabling problem in the literature, our model has some advantageous points. The first feature of our model is to define event base decision variables and constraints. These variables make it possible to use them in decompositions or relaxation algorithms so that each sub-problem consists of a decision making problem related to each event. Constraint (12) which integrates the decision variables allows the occurrence of only one event and creates a suitable situation for decomposition. In this situation a difficult problem can be decomposed to some simpler sub-problems and increases the possibility of solving real size problems.

On the other hand, as another benefit of the model, planning of the station capacity is simultaneously possible in our timetabling model. Constraints (14) and (16) make it possible to consider the capacity of stations and sidings. Most of models in the literature decompose the problem into two separate problems. One to define and solve a general timetable and another to deal with the complex station capacity planning problem $[2,5,10,12,14,37,42-44]$. The station capacity planning problem needs special attentions, so some research studies and algorithms in recent years have been dedicated to this field of study [21,45-51]. Based on this point of view, the proposed model integrates the two models and provides an integrated answer. Moreover, the new definition of decision variables classifies the solution space and gives the possibility of defining and removing the areas of solution space that are not rationally as part of the optimal solution area. A comprehensive investigation of this topic will be as follows.

\subsection{Complexity analysis}

Although the problem is generally considered as a NPhard one, almost every paper considers a different version of the problem. For this reason, we give a NP-hardness proof for the specific problem considered. Our proof will show that our problem is a reduction of $F F_{s} \mid r_{J} \sum C_{j}$ In order to show the complexity of the problem, we used a reduction from a flexible flowshop model. Our proposed model is a complicated version of a flexible flow-shop model with capacitated buffer and some limitations on the job assignment to machines. According to the definition of flow-shop problem by [52], there are $m$ machines in series and each job passes each machine in the same route. If the number of identical machines in at least one stage be greater than 1, the problem is classified as a Flexible Flow Shop problem $\left(F F_{s}\right)$. Moreover, when machine $M$ in a specific stage is not capable of processing alljobs, $M_{j}$ defines this machine eligibility constraint. Finally, if there is a limited buffer between the two machines in a way that when the buffer is full, the machine is not allowed to release a new job and the block constraint obligates the completed job to remain in the previous machine.

To further illustrate the issue, consider the trains as jobs and the stations as machines in which each job (train) must pass through machines (stations); as Zhou and Zhong [10] and Burdett and Kozan [20] have proposed. Based on the definition of flow-shop problem by Pinedo [52], this problem can be classified as flow-shop problem with block constraint. Moreover, the number of platforms in each station or siding defines the capacity of the station or siding that may be greater than one, so the problem is classified as a flexible flow-shop problem or multi-stage parallel machine problem [20]. On the other hand, some express trains are scheduled to not stop in some stations and the stations also have some specific platforms for non-stopping trains. This situation defines the machine's eligibility constraint. Furthermore, there are intrinsically some limitations on the start time of each train. Thus, the problem can be shown as $F F_{s} \mid M_{J}, r_{J}$, block $\mid \gamma$ according to the notation of [52]. Since the problem of $F F_{s} \| \sum C_{j}$ is NP-hard [53], our problem is NP-hard. Therefore, it is not possible to solve large-scale problems in a poly-nominal time and some policies should be conducted to reduce the complexity of the problem.

\section{Upper bound rules}

In this section, we define some rules to efficiently limit the number of binary variables and also create efficient upper bounds. Binary variables, which increase the complexity of the problem, are used to identify the sequencing and overtaking's details of trains. Therefore, decreasing the number of binary variables decreases the number of decisions and make the problem easier to solve. There exist some studies in order to limit the number of unnecessary overtaking cases. Burdett and Kozan [18] introduced some algorithms to identify and correct some conflicts such as multiple overtaking and compound moves. In their article, they restricted trains to multiple overtakings, and there is no point in restricting the general situations of overtaking. In the following, we propose some general rules to restrict undesirable overtaking among the trains in a network. We use trains' parameters in order to define usefulness or undesirability of overtaking among every two trains. 
In each railway network, some trains have the same characteristics such as travel and dwell times. It is clear that the study of this group of trains can lead to rules that may relax the binary decision variables among the similar trains. For example, in a German railway network, there are eight types of train, such as Intercity Express (ICE), Regional Bahn (RB) and S-Bahn (S), and most trains in each group have the same parameters in the network. Consider two trains $A$ and $B$ in a group with exactly the same speed and dwell time parameters in all network stations. The idea is that overtaking each of these two trains from each other is not beneficial. Consider the timetable in which train $A$ is scheduled to overtake train $B$ in station $s$. Because the dwell and travel times of the two trains are identical, train $B$ has to delay at the station, and as a result, the objective function increases. By this idea, we try to expand the simple rule to the whole of the trains, and we will obtain the rules that fix unnecessary overtaking binary variables considering the general situations. In a word, we want to obtain the situations among every two trains where occurrence of Events 1 and 2 may result in a delay in the timetable. We consider two random trains and the objective functions of the two timetables in order to find the situations and related rules that overtaking is not beneficial. We compare the objective functions of the timetable in which trains go ahead without overtaking with those of the timetable in which one train overtakes another. In these investigations, we assume that there is no deliberate delay. The results of the investigations can be used in the reduction of binary variables and the CPU time.

Now, assume two trains $t^{\prime}$ and $t$, which are now in stations $s$ and $q(=s+1)$, consecutively, as shown in Figure 3, in which station $q$ has two platforms. In Figure 3, the two horizontal lines show the scheduling of the related stations. Points $k$ and $w$ define the arrival and departure times of train $t$ in station $q$, respectively. The time interval between $R$ and $K$ represents travel time of train $t$ between two consecutive stations $s$ and $q$. Also, the time interval between $K$ and $W$ defines the dwell time of train $t$ in station $q$.

At first, we define two time intervals in order to

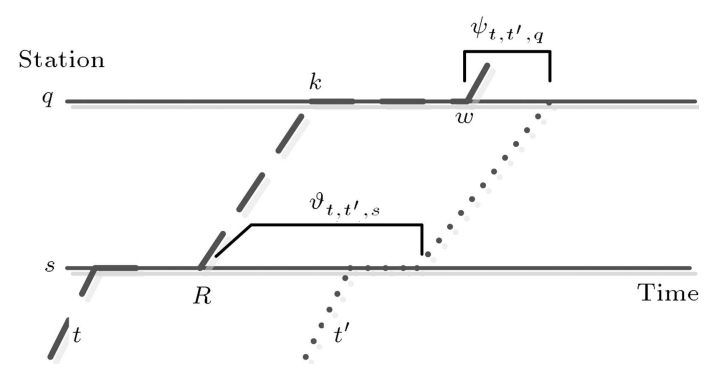

Figure 3. The situation of trains $t$ and $t^{\prime}$ in stations $s$ and $q$. characterize different situations. We define $\Psi_{t, t^{\prime}, q}$ as the time interval between the departure time of train $t$ from station $q$ and the arrival time of train $t^{\prime}$ to this station, i.e. $\Psi_{t, t^{\prime}, q}=S_{t l^{\prime} q}-C_{t l q}$. Also, $\vartheta_{t, t^{\prime}, s}$ is the time interval between the departure times of two trains from station $s$, i.e. $\vartheta_{t, t^{\prime}, s}=C_{l t^{\prime} s}-C_{l t s}$. If $\Psi_{t, t^{\prime}, q}$ be greater than zero, the overtaking of train $t^{\prime}$ from train $t$ in station $q$ is not recommended, because overtaking will result in a delay for train $t$. So, the timetable without overtaking is selected for the station and trains. Moreover, without loss of generality, we can assume that $\vartheta_{t, t^{\prime}, s}$ is greater than zero. The mentioned condition can be written as follows:

$$
\text { If } 0<\Psi_{t, t^{\prime}, q} \text { and } 0<\vartheta_{t, t^{\prime}, s} \text { then } x_{t, t^{\prime}, s}=0 \text {. }
$$

In some situations, this rule does not give the optimal solution, although it fixes lots of binary variables. To investigate further, we analyze the situation where the mentioned condition is not satisfied, i.e. two trains are simultaneously in the station and one train can overtake another, meaning that $\Psi_{t, t^{\prime}, q}$ is lower than zero. This condition is met when the time interval between the arrival and departure of two trains in station $q$ is lower than the required station safety time. The first derived condition is:

$$
\begin{aligned}
& \psi_{t t^{\prime} q} \leq 0 \equiv S_{l t^{\prime} q} \leq C_{l t q}+S F_{l, q}, \\
& C_{l t^{\prime} s}+\underline{T}_{l, s, q}^{t^{\prime}} \leq S_{l t^{\prime} q} \leq\left(C_{l t s}+\underline{T}_{l . s, q}^{t}+\underline{D}_{l, q}^{t}\right)+S F_{l, q},
\end{aligned}
$$

$$
\equiv C_{l t^{\prime} s}-C_{l t s} \leq \underline{T}_{l, s, q}^{t}-\underline{T}_{l, s, q}^{t^{\prime}}+\underline{D}_{l, q}^{t}+S F_{l, q} .
$$

In Eq. (20), it is obvious that the departure time of train $t$ from station $q$ is equal to its departure time from station $s$ plus its travel time between the two stations and its dwell time in station $q$, i.e. $C_{l t q}=$ $C_{l t s}+\underline{T}_{l, s, q}^{t}+\underline{D}_{l, q}^{t}$. Also, as another fact, the arrival time of train $t^{\prime}$ to station $q$ is greater than its departure time from station $s$ and the travel time between the two stations, i.e. $S_{l t^{\prime} q} \geq C_{l t^{\prime} s}+\underline{T}_{l, s, q}^{t^{\prime}}$. On the other hand, it is obvious that $\vartheta_{t, t^{\prime}, s}$ is greater than dwell time of train $t^{\prime}$ in station $s$ plus safety time of this station, i.e., $\underline{D}_{l, s}^{t^{\prime}}+S F_{l, s} \leq C_{l t^{\prime} s}-C_{l t s}$. Thus, we can write:

$$
\begin{aligned}
\underline{D}_{l, s}^{t^{\prime}} & +S F_{l, s} \leq C_{t^{\prime} s}-C_{t s} \\
& \leq \underline{T}_{l, s, q}^{t}-\underline{T}_{l, s, q}^{t^{\prime}}+\underline{D}_{l, q}^{t}+S F_{l, q} .
\end{aligned}
$$

The derived condition in Eq. (22) describes the situation where the two trains are simultaneously in station $q$, so that overtaking may be beneficial, and we will investigate it later on. On the other hand, if the condition in Eq. (22) is not met (like the situation that is shown in Figure 3), overtaking train $t^{\prime}$ from train $t$ in station $q$ results at least in $\underline{D}_{l, s}^{t^{\prime}}+S F_{l, s}-\left(\underline{T}_{l, s, q}^{t}-\right.$ 
$\left.\underline{T}_{l, s, q}^{t^{\prime}}+\underline{D}_{l, q}^{t}+S F_{l, q}\right)$ obligatory delay for train $t$. This situation causes the increasing of objective function and a grasp vision Constraint (23) can be added to the problem under the following conditions:

$$
\begin{aligned}
& \forall \in L ; \quad \forall s, q \in S_{l} ; \quad \forall t \neq t^{\prime} \in T_{l}, \\
& \underline{D}_{l, s}^{t^{\prime}}+S F_{l, s}>\underline{T}_{l, s, q}^{t}-\underline{T}_{l, s, q}^{t^{\prime}}+\underline{D}_{l, q}^{t}+S F_{l, q},
\end{aligned}
$$

and the constraint is as follows that forbids overtaking two trains in station $s$ :

$$
x_{t, t^{\prime}, s} \leq 0 \text {. }
$$

In a specific condition, the added constraint brings the optimal solution as follows:

Proposition 1: Suppose that there is a railway network with three stations and no opposite direction trains. Furthermore, there are two consecutive trains, $t$ and $t^{\prime}$, that in the first station, train $t$ precedes train $t^{\prime}$. Train $t$ will finish its path first if $\vartheta_{t, t^{\prime}, 1}$ is greater than $\Psi_{t, t^{\prime}, 2}$, i.e. the time interval between the departure times of the two trains in the first station $\left(\vartheta_{t, t^{\prime}, 1}\right)$ is greater than the time interval between the arrival time of train $t^{\prime}$ to the second station and the departure time of train $t$ from the second station $\left(\Psi_{t, t^{\prime}, 2}\right)$.

Proof: As described in the proposition, there are three stations and the only station where overtaking is possible is the second station. Moreover, as described in the proposition, the condition in Eq. (22) is not satisfied. If train $t^{\prime}$ overtakes train $t$ in the second station, as shown in Eq. (22), the objective function at least increases by $\underline{D}_{l, s}^{t^{\prime}}+S F_{l, s}-\left(\underline{T}_{l, s, q}^{t}-\left(\underline{T}_{l, s, q}^{t^{\prime}}+\right.\right.$ $\left.\underline{D}_{l, q}^{t}+S F_{l, q}\right)$. So, the optimal route is the current route where train $t$ ends its route first.

On the other hand, we consider the situation that the condition in Eq. (22) is met, i.e. $\Psi_{t, t^{\prime}, q}<0$ and $0<\vartheta_{t, t^{\prime}, s}$; in a word, $\underline{D}_{l, s}^{t^{\prime}}+S F_{l, s} \leq \underline{T}_{l, s, q}^{t}-$ $\underline{T}_{l, s, q}^{t^{\prime}}+\underline{D}_{l, q}^{t}+S F_{l, q}$. In this situation, train $t^{\prime}$ arrives in station $q$ before train $t$ departs from the station. In order to simplify the investigation and also make the equations more clear, we try to classify different circumstances and then check their conditions. As the first classification factor, we consider the relation between $\Psi_{t, t^{\prime}, q}$ and $S F_{l, q}$. In the situation where Eq. (22) is valid, we consider the condition that $\left|\Psi_{t, t^{\prime}, q}\right|$ is lower than the station's safety time, i.e. $\Psi_{t, t^{\prime}, q}<$ $S F_{l, q}$, so train $t^{\prime}$ will be delayed until the safety time is met. With regard to this condition, two different situations in which the safety time can/cannot be satisfied will be discussed. In order to obtain the relation between $\Psi_{t, t^{\prime}, q}$ and $S F_{l, q}$ more clearly, we use equation $\psi_{t, t^{\prime}, q}=C_{l t^{\prime} s}+\underline{T}_{l, s, q}^{t^{\prime}}-C_{l t s}-\underline{T}_{l, s, q}^{t}$ which describes the time interval between the arrival times of the two trains to station $q$, and according to inequality, $\underline{D}_{l, s}^{t^{\prime}}+S F_{l, s} \leq C_{t^{\prime} s}-C_{t s}$ is written as $\psi_{t, t^{\prime}, q}=$ $S F_{l, s}+\underline{D}_{l, s}^{t^{\prime}}+\underline{T}_{l, s, q}^{t^{\prime}}-\underline{T}_{l, s, q}^{t}$. So, the first classification can be written as $S F_{l, s}+\underline{D}_{l, s}^{t^{\prime}}+\underline{T}_{l, s, q}^{t^{\prime}}-\underline{T}_{l, s, q}^{t} \leq S F_{l, q}$ or $S F_{l, s}+\underline{D}_{l, s}^{t^{\prime}}+\underline{T}_{l, s, q}^{t^{\prime}}-\underline{T}_{l, s, q}^{t}>S F_{l, q}$.

Moreover, as the second classification factor, we consider the situation where train $t^{\prime}$ overtakes train $t$ in station $q$ which causes scheduled delays for train $t$. In another situation where there is not any scheduled delay for train $t$, the time interval in which train $t$ stops in station $q$ is greater than the required safety time for train $t^{\prime}$ to enter station $q\left(S F_{l, q}\right)$ plus its dwell time in that station $\left(\underline{D}_{l, q}^{t^{\prime}}\right)$ plus another required safety time $\left(S F_{l, q}\right)$ after train $t^{\prime}$ departs from station $q$. So, $S F_{l, q}+\underline{D}_{l, q}^{t^{\prime}}+S F_{l, q} \geq \underline{D}_{l, q}^{t}$ and $S F_{l, q}+\underline{D}_{l, q}^{t^{\prime}}+$ $S F_{l, q}<\underline{D}_{l, q}^{t}$ are the two classifications. According to these two classifications, four different conditions are obtained, and their characteristics and assumptions are summarized in Eq. (24). Also, one example from each of them is shown in Figure 4. For all $l \in L, \quad \forall t \neq$ $t^{\prime} \in T_{l}$, and $\forall s, q \in S_{l}$, the conditions are calculated as shown in Box I.

Similar to Proposition 1, for each condition, we try to find the situations where overtaking is not beneficial. So, we should compare the objective functions of the two timetables that, firstly, train $t^{\prime}$ overtakes train $t$ and, secondly, the situation where no overtaking occurs. In these comparisons, the aim is to find the relation of the parameters in a way that overtaking by a grasp view results in increasing of the objective function.

Therefore, we obtain the objective function of the timetable in which train $t$ arrives in and departs

$$
\underline{D}_{l, s}^{t^{\prime}}+S F_{l, s} \leq \underline{T}_{l, s, q}^{t}-\underline{T}_{l, s, q}^{t^{\prime}}+\underline{D}_{l, q}^{t}+S F_{l, q} \begin{cases}S F_{l, s}+\underline{D}_{l, s}^{t^{\prime}}+\underline{T}_{l, s, q}^{t^{\prime}}-\underline{T}_{l, s, q}^{t} \leq S F_{l, q} & \left\{\begin{array}{l}
\underline{D}_{l, q}^{t^{\prime}}+2 S F_{l, q} \geq \underline{D}_{l, q}^{t}(1) \\
\underline{D}_{l, q}^{t^{\prime}}+2 S F_{l, q}>\underline{D}_{l, q}^{t}(2)
\end{array}\right. \\
S F_{l, s}+\underline{D}_{l, s}^{t^{\prime}}+\underline{T}_{l, s, q}^{t^{\prime}}-\underline{T}_{l, s, q}^{t} \leq S F_{l, q} & \left\{\begin{array}{l}
\underline{D}_{l, q}^{t^{\prime}}+2 S F_{l, q} \geq \underline{D}_{l, q}^{t}(3)(24) \\
\underline{D}_{l, q}^{t^{\prime}}+2 S F_{l, q} \geq \underline{D}_{l, q}^{t}(4)
\end{array}\right.\end{cases}
$$



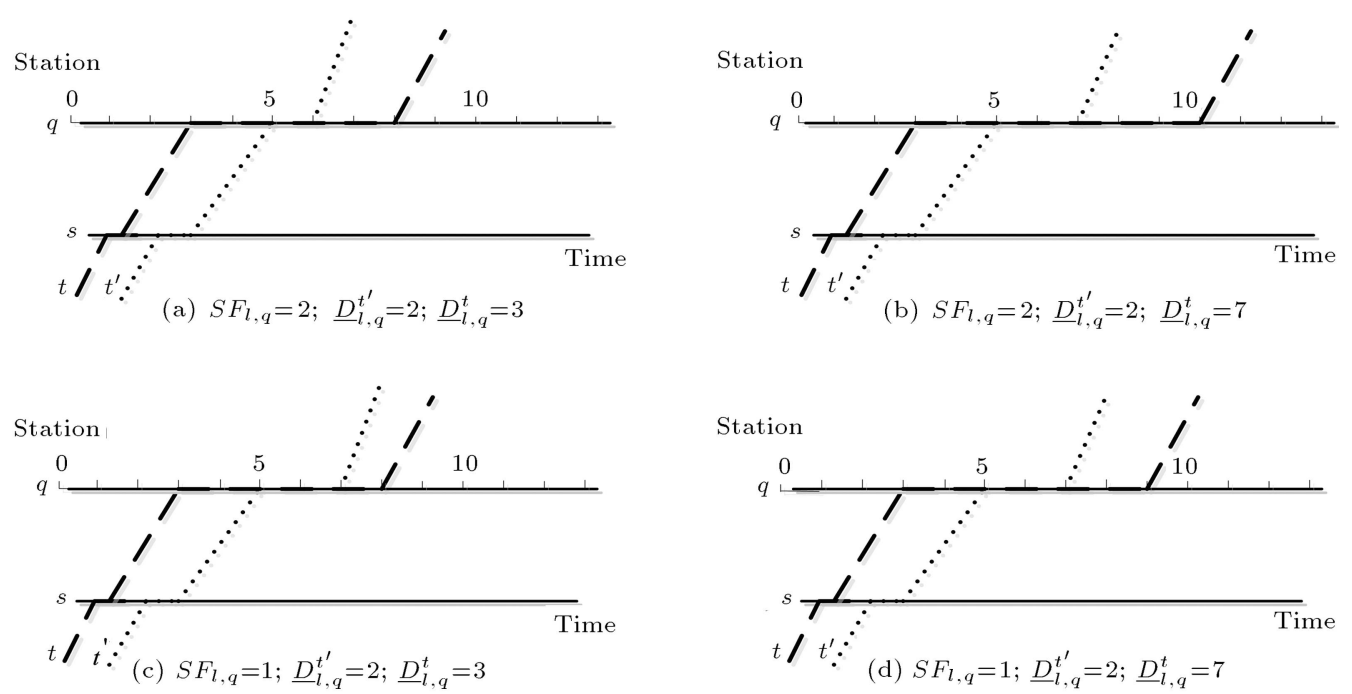

Figure 4. Four conditions in obtaining upper-bound (a), (b), (c), and (d), respectively, define the first, second, third, and fourth defined conditions.

from stations first. We will use this amount in each of the four conditions in order to make comparisons. Then, we calculate the objective function in each of the mentioned classified conditions in a way that train $t^{\prime}$ overtakes train $t$ in station $q$. These objective functions will be used in the mentioned comparison in order to obtain some heuristic rules. In the following three equations, we calculate the objective function of the no-overtaking situation.

$$
\begin{aligned}
C_{l t q} & =C_{l t s}+\underline{T}_{l, s, q}^{t}+\underline{D}_{l, q}^{t}, \\
C_{l t^{\prime} q} & =C_{l t s}+\left(C_{l t^{\prime} s}-C_{l t s}\right)+\underline{T}_{l, s, q}^{t^{\prime}} \\
& +\left(\underline{T}_{l, s, q}^{t}-\underline{T}_{l, s, q}^{t^{\prime}}+\underline{D}_{l, q}^{t}+S F_{l, q}\right. \\
& \left.-\left(C_{l t^{\prime} s}-C_{l t s}\right)\right)+\underline{D}_{l, q}^{t^{\prime}}, \\
C_{l t q} & +C_{l t^{\prime} q}=2 C_{l t s}+2 \underline{T}_{l, s, q}^{t}+2 \underline{D}_{l, q}^{t} \\
& +S F_{l, q}+\underline{D}_{l, q}^{t^{\prime}} .
\end{aligned}
$$

Eqs. (25) and (26) respectively show the departure times of trains $t$ and $t^{\prime}$ from station $q$, and Eq. (27) is the summation of the two departure times. In Eq. (25), $C_{l t q}=C_{l t s}+\underline{T}_{l, s, q}^{t}+\underline{D}_{l, q}^{t}$ defines the departure time of train $t$ from station $q$ without any delay. In Eq. (26), the departure time of train $t^{\prime}$ consists of the departure time from station $s$, the travel time between the two stations, the amount of time that train $t^{\prime}$ must be delayed until train $t$ departs from station $q$, i.e. $\underline{T}_{l, s, q}^{t}$ $\underline{T}_{l, s, q}^{t^{\prime}}+\underline{D}_{l, q}^{t}+S F_{l, q}-\left(C_{l t^{\prime} s}-C_{l t s}\right)$ and its dwell time in station $q$. The scheduled delay amount was obtained in Eq. (22). As shown in Eq. (27), the result is $C_{l t q}+C_{l t^{\prime} q}=2 C_{l t s}+2 \underline{T}_{l, s, q}^{t}+2 \underline{D}_{l, q}^{t}+S F_{l, q}+\underline{D}_{l, q}^{t^{\prime}}$ and will be used later for the comparisons.

On the other hand, we have to calculate the objective function of the timetable where overtaking occurs for each of the mentioned conditions. Regarding the first set of assumptions in Eq. (24), the departure time of the two trains is calculated in the situation where train $t^{\prime}$ overtakes train $t$ in station $q$. The departure times of trains $t, t^{\prime}$ are shown in Eqs. (28) and (29), respectively, and the objective function is as Eq. (30). In order to clarify the origin of the equations, the elements of each departure time are described below:

Departure time of train $t=$ its arrival time to station $q+$ safety time of station $q+$ dwell time of train $t^{\prime}$ in station $q+$ safety time of station;

Departure time of train $t^{\prime}=$ its departure time from station $s+$ travel time among two stations $s$ and $q+$ obligatory delay to insure the safety time of station $q+$ dwell time of train $t^{\prime}$ in station $q$.

$$
\begin{aligned}
C_{l t q}= & \left(C_{l t s}+\underline{T}_{l, s, q}^{t}\right)+S F_{l, q}+\underline{D}_{l, q}^{t^{\prime}}+S F_{l, q}, \\
C_{l t^{\prime} q}= & \left(C_{l t s}+\left(C_{l t^{\prime} s}-C_{l t s}\right)\right)+\left(\underline{T}_{l, s, q}^{t^{\prime}}+S F_{l, q}\right. \\
& \left.-\left(C_{l t^{\prime} s}+\underline{T}_{l, s, q}^{t^{\prime}}-C_{l t s}-\underline{T}_{l, s, q}^{t}\right)\right), \\
C_{l t q}+ & C_{l t^{\prime} q}=2 C_{l t s}+2 \underline{T}_{l, s, q}^{t}+3 S F_{l, q}+2 \underline{D}_{l, q}^{t^{\prime}} .
\end{aligned}
$$

By comparing Eqs. (27) and (30), Eq. (32) shows the relation of parameters in which overtaking is not recommended and the objective function of the timetable with overtaking is greater than the one without overtaking. Eq. (31) shows the details of the comparison: 


$$
\begin{aligned}
2 C_{l t s}+ & 2 \underline{T}_{l, s, q}^{t}+2 \underline{D}_{l, q}^{t}+S F_{l, q}+\underline{D}_{l, q}^{t^{\prime}} \\
& \leq 2 C_{l t s}+2 \underline{T}_{l, s, q}^{t}+3 S F_{l, q}+2 \underline{D}_{l, q}^{t^{\prime}} \\
2 \underline{D}_{l, q}^{t} & \leq \underline{D}_{l, q}^{t^{\prime}}+2 S F_{l, q} .
\end{aligned}
$$

Thus, in the circumstance where the set of assumptions of the first condition is true and inequality (32) is valid, we can propose that overtaking is not a good decision and we recommend adding Constraint (33) to the problem under the following conditions:

$$
\begin{aligned}
& \forall t \neq t^{\prime} \in T_{l} ; \quad \forall l \in L ; \quad \forall s, q \in S_{l} \\
& \underline{D}_{l, s}^{t^{\prime}}+S F_{l, s} \leq \underline{T}_{l, s, q}^{t}-\underline{T}_{l, s, q}^{t^{\prime}}+\underline{D}_{l, q}^{t}+S F_{l, q}, \\
& S F_{l, s}+\underline{D}_{l, s}^{t^{\prime}}+\underline{T}_{l, s, q}^{t^{\prime}}-\underline{T}_{l, s, q}^{t} \leq S F_{l, q}, \\
& 2 S F_{l, q}+\underline{D}_{l, q}^{t^{\prime}} \geq \underline{D}_{l, q}^{t}, \\
& 2 \underline{D}_{l, q}^{t} \leq 2 S F_{l, q}+\underline{D}_{l, q}^{t^{\prime}},
\end{aligned}
$$

and the constraint is in the following which forbids the overtaking of two trains in station $s$ :

$$
x_{t, t^{\prime}, s} \leq 0
$$

By a similar approach, the derived equation in condition (2) is $\underline{D}_{l, q}^{t^{\prime}} \leqslant 0,0 \leq \underline{D}_{l, q}^{t^{\prime}}+S F_{l, q}$ in Condition (3), and $\underline{D}_{l, q}^{t} \leq \underline{D}_{l, q}^{t^{\prime}}$ in Condition (4). Related constraints are described in Eqs. (34) to (36), respectively. For all $l \in L, \forall t \neq t^{\prime} \in T_{l}, \forall s, q \in S_{l}$ and in the condition where the parameter of the problem satisfies $\underline{D}_{l, s}^{t^{\prime}}+S F_{l, s} \leq \underline{T}_{l, s, q}^{t}-\underline{T}_{l, s, q}^{t^{\prime}}+\underline{D}_{l, q}^{t}+S F_{l, q}$ the constraints are as follows:

$$
\begin{aligned}
& x_{t, t^{\prime}, s} \leq 0 ; \quad S F_{l, s}+\underline{D}_{l, s}^{t^{\prime}}+\underline{T}_{l, s, q}^{t^{\prime}}-\underline{T}_{l, s, q}^{t} \leq \mathrm{S} F_{l, q} \\
& 2 \mathrm{~S} F_{l, q}+\underline{D}_{l, q}^{t^{\prime}}<\underline{D}_{l, q}^{t} ; \quad \underline{D}_{l, q}^{j} \leq 0 \\
& x_{t, t^{\prime}, s} \leq 0 ; \quad S F_{l, s}+\underline{D}_{l, s}^{t^{\prime}}+\underline{T}_{l, s, q}^{t^{\prime}}-\underline{T}_{l, s, q}^{t}>S F_{l, q} \\
& 2 S F_{l, q}+\underline{D}_{l, q}^{t^{\prime}} \geq \underline{D}_{l, q}^{t} ; \quad 0 \leq \underline{D}_{l, q}^{t^{\prime}}+S F_{l, q} \\
& x_{t, t^{\prime}, s} \leq 0 ; \quad S F_{l, s}+\underline{D}_{l, s}^{t^{\prime}}+\underline{T}_{l, s, q}^{t^{\prime}}-\underline{T}_{l, s, q}^{t}>S F_{l, q} \\
& 2 S F_{l, q}+\underline{D}_{l, q}^{t^{\prime}}<\underline{D}_{l, q}^{t} ; \quad \underline{D}_{l, q}^{t} \leq \underline{D}_{l, q}^{t^{\prime}}
\end{aligned}
$$

As stated before, these rules are able to fix some binary variables. Thus, by the reduction of binary variables, a solution in a shorter time can be obtained; however, there is no guarantee about its optimality and it can act as an upper bound for the problem. In addition, if we consider the trains in a group and that they have the same travel and dwell times, phrase $\underline{T}_{l, i, s, s+1}-\underline{T}_{l, j, s, s+1}$ will relax and the mentioned conditions for each constraint will simplify. Furthermore, the dwell times for most of the stations in a network are equal and can be relaxed from some of the constraints. Only in the stations where there is a crossing point of two or more lines, dwell time is slightly different from those of other stations. In addition, the safety time, which is based on the geographical specifications of a station and the line, is usually equal for stations. Thus, the mentioned constraints are simplified for most trains and stations so that they are able to decrease the complexity of the problem.

\section{Lower bound}

In this section, we present a Lagrangian Relaxation (LR) lower bound algorithm to estimate a powerful lower bound of the objective function. The Lagrangian Relaxation algorithm is one of the most efficient algorithms, obtaining lower bound. In this algorithm, complex constraints are relaxed from the set of constraints and are added to the objective function with a penalty multiplier. Selecting the relaxed constraints with a non-zero integrality gap and updating the multiplier of relaxed constraint are of high importance [54]. Here, the overtaking constraints and capacity-related constraints increase the complexity of the problem. The binary decision variables are the elements which increase the complexity of the constraints. Constraint (12) binds all the binary decision variables, and it seems that it is the most difficult constraint in the current set. Thus, Constraint (12), which integrates the events together, is the best candidate, satisfies the mentioned criteria, and should be selected so that the problem can be efficiently relaxed. Considering the selected constraint, the objective function of LR model is as follows:

$$
\begin{aligned}
\min z= & \sum_{l=1}^{L} \sum_{t=1}^{T} c_{l t m}+\sum_{l \in L_{E}} \sum_{t \in T_{l}} \sum_{t^{\prime} \in T_{l} ; t \neq t^{\prime}} \sum_{s \in S_{l}} \\
& u_{l, t, t^{\prime}, s}^{k}\left(x^{\prime \prime}{ }_{t, t^{\prime}, s}+x^{\prime}{ }_{t, t^{\prime}, s}+x_{t, t^{\prime}, s}+x_{t^{\prime}, t, s}-1\right),
\end{aligned}
$$

where $u_{l, t, t^{\prime}, s}^{k}$ represents the Lagrangian multipliers and the sub-gradient method updates them because of its efficacy [55-57]. Other constraints, except Constraint (12), are embedded in the LR model. The multipliers are initially interpreted as the price (marginal cost) of the relaxed constraint in a feasible solution. Because the constraint is in the equality form, the multipliers are always zero in a feasible solution. The multipliers are iteratively adjusted using the result of the model in a way that helps to improve the amount of the lower bound. Therefore, in each step, the amount of $x_{t, t^{\prime}, s}^{\prime \prime}+$ $x_{t, t^{\prime}, s}^{\prime}+x_{t, t^{\prime}, s}+x_{t^{\prime}, t, s}-1$ is calculated and called as 
$\gamma_{l, t, t^{\prime}, s}$, and it is used to update the multipliers. Eqs. (38) to (40) demonstrate this process as a sub-gradient method:

$$
\gamma_{l, t, t^{\prime}, s}=x_{t, t^{\prime}, s}^{\prime \prime}+x_{t, t^{\prime}, s}^{\prime}+x_{t, t^{\prime}, s}+x_{t^{\prime}, t, s}-1
$$

Step_Size

$$
\begin{gathered}
\leq \theta \frac{\left(U B-\text { Objective }_{k}\right)}{\sum_{l \in L_{E}} \sum_{t \in T_{l}} \sum_{t^{\prime} \in T_{l} ; t \neq t^{\prime}} \sum_{s \in S_{l}}\left(\gamma_{l, t, t^{\prime}, s}^{2}\right)} \\
u_{l, t, t^{\prime}, s}^{k+1}=u_{l, t, t^{\prime}, s}^{k}+\gamma_{l, t, t^{\prime}, s} \times \text { Step_Size } k
\end{gathered}
$$

where $k$ is the iteration index used in the LR model, UB is the objective function of a feasible solution, "Objective ${ }_{k}$ " is the amount of the objective function of the LR model in iteration $k$, and $\theta$ is a parameter by an initial value as 2 . Eq. (38) defines the amount in which the relaxed constraint is not satisfied. Eq. (39) calculates the amount of the step-size parameter in iteration $k$ and Eq. (40) updates the amount of Lagrangian multipliers for the next iteration. Using these parameters, the LR iteratively updates the parameters to obtain the optimal solution. The related procedure is shown in Figure 5.

At the first step, in order to define UB, which is used in Eq. (39), we find a feasible solution in which trains go through the stations with a lexicographical order and without any overtaking. The objective function of this solution multiplied by 1.05 defines UB. Moreover, we get the objective function of the problem with relaxed binary variables. The objective function of this Relaxed Mixed Integer Problem (RMIP) will be used as a benchmark for calculating the improvement of the LR model. After the first iteration, the parameters and multipliers will update and the next iteration will start until one of these conditions is met:

- $\max _{l, t, t^{\prime}, s}\left(u_{l, t, t^{\prime}, s}^{k+1}-u_{l, t, t^{\prime}, s}^{k}\right)<0.005$;

- Number of iterations exceeds 100.

Moreover, in each step, if the objective function of LR model does not increase, the amount of $\theta$ will be halved, which helps it to increase the lower bound.

\section{Experimental results}

In order to demonstrate the computational efficiency of the mathematical programming model and the proposed upper and lower bounds' rules, a series of numerical experiments are illustrated. The following case study is based on the real parameters of Tehran Metro line 5 between Tehran and Golshahr with 12 stations and 40 kilometers length, in which its general view is magnified in Figure 6. The actual TehranGolshahr line is a double-tracked line, in which every 11-minute trains run from Tehran to Golshahr, and vice versa. There are some express trains that stop only in three stations and have no dwell time in other stations. The travel time for the express trains is about 32 minutes and it is 52 minutes for other trains.

Each station has at least one platform in which the loading and unloading of passengers occur. Except for the first and last stations, the main line of each station has no platform for passengers to board or alight from trains. Trains can overtake each other in the sidings or in the stations.

There are different headways in the network in a day. The network headway in peak hours alters and the minimum amount of the departure time between two trains reaches 480 seconds. Regarding the complexity of timetabling in the peak-hours, we consider the related parameters of the peak-hours to obtain a timetable.

The computational time of train timetabling algorithms can be affected by a number of siding and station's capacities, number of trains, number of stations and sidings, and the variability of trains' parameters. In the following numerical experiments, we focus on the impact of the variability of station capacity, trains and number of trains, since these factors mainly influence the structure of a train timetable and the resulting number of possible solutions.

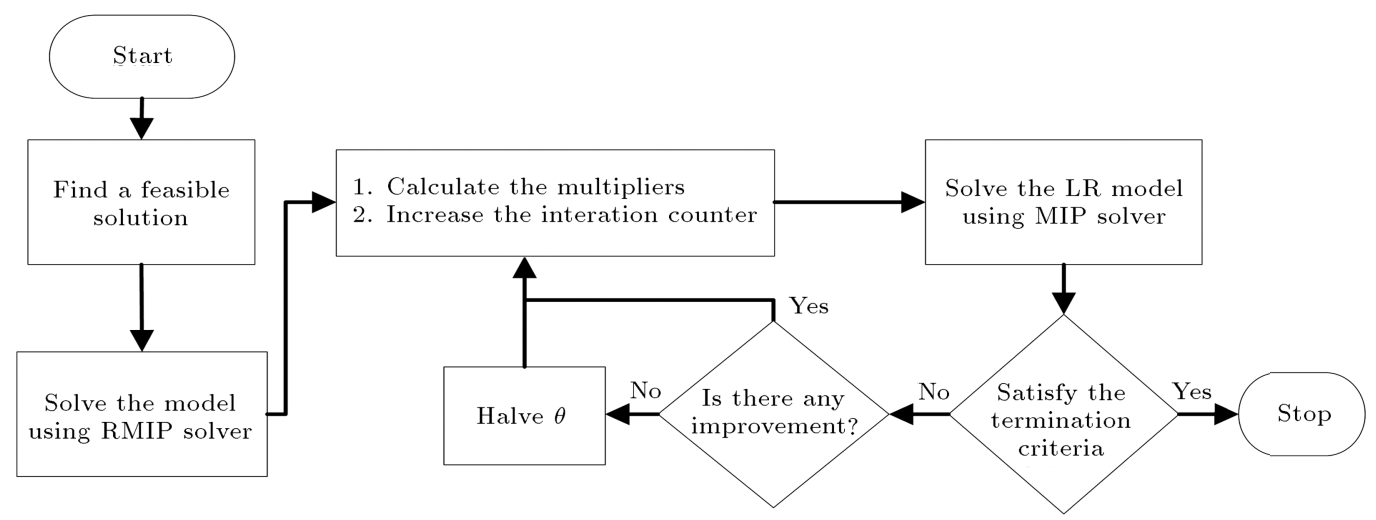

Figure 5. Lagrangian relaxation algorithm procedure. 


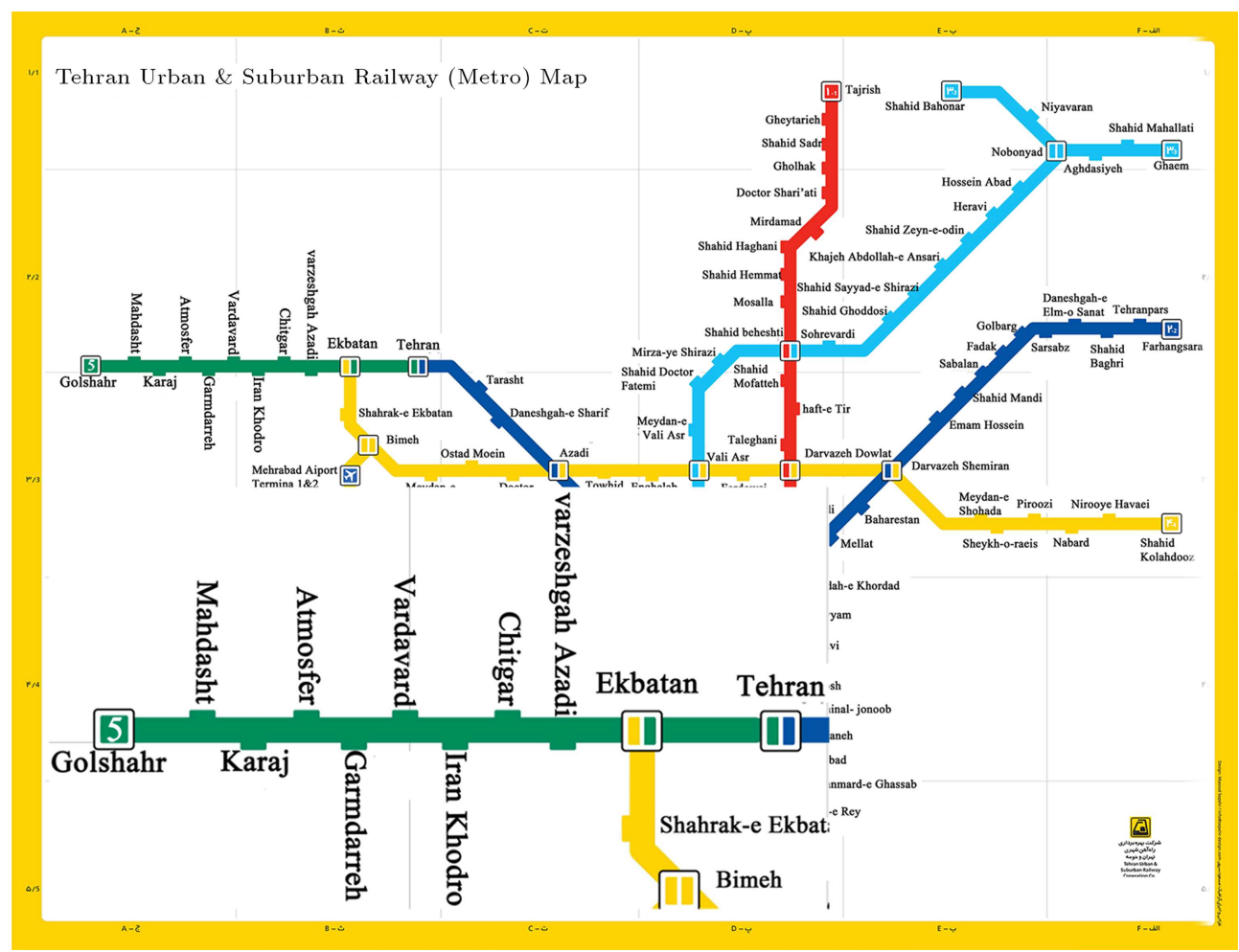

Figure 6. General view of the Tehran Metro and Line 5.

However, the real-world problem used in this study only offers two types of trains, and a fixed number of trains that creates a simple problem to solve. To allow a comprehensive and systematic assessment, we construct random instances to evaluate the performance of the proposed algorithm. In this way, we increased the capacity of some middle stations. The stations were chosen randomly and the number of facilities increased randomly up to three. As another factor for increasing the complexity of the problem, we increased the variability of the travel time for some trains in a way that we increased the travel time up to $100 \%$ for all stations for $50 \%$ of randomly selected trains.

In the following experiments, consciousness and authenticity of the model, the effectiveness of the heuristic upper bound rules, and the quality of the proposed Lagrangian Relaxation method are investigated. In these investigations, five problems with different number of trains have been solved with the proposed models. We have used GAMS workstation and CPLEX solver to solve the problems on a PC equipped with 3 $\mathrm{GHz}$ sixteen cores processor and $18 \mathrm{~GB}$ of RAM.

Figure 7 illustrates the resulting optimal timetable for nine trains for the case of TehranGolshahr line. As shown in the Atmosfer station, three trains 3, 4, and 5 are in the station simultaneously; in stations Vardavard and Garmdareh, train 3 overtakes trains 5 and 4 , and the other general constraints of the problem are satisfied.

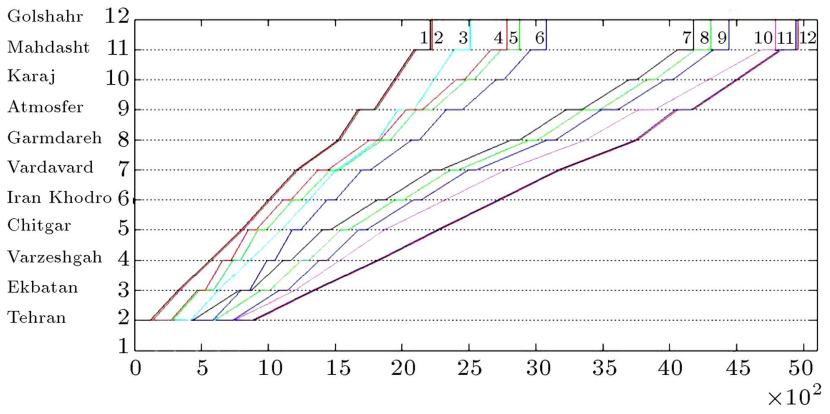

Figure 7. Time-distance graph for 12 trains and six last stations.

\subsection{Performance of upper-bound rules}

In order to analyze the five introduced upper-bound rules, we solved each problem by GAMS using the CPLEX solver, as an efficient and exact solver, and the optimal solutions were gathered as a benchmark. The optimal solutions are shown in Table 1. The quality of the rules is measured by percentage gap between the obtained upper bound and the corresponding optimal value. In addition, improvement in CPU time is the second most important criterion for analyzing the obtained rules.

In order to analyze the effectiveness of upperbound rules, the obtained constraints (Constraints (23), (33)-(36)) are embedded in the problem. This problem is named as Upper-Bound Rule Problem (UBRP) in the results and hereafter. The objective 
Table 1. The comparison result of CPLEX and UBRP.

\begin{tabular}{lcccccccc}
\hline & & \multicolumn{7}{c}{ Number of trains } \\
\cline { 3 - 9 } & & $\mathbf{6}$ & $\mathbf{7}$ & $\mathbf{8}$ & $\mathbf{9}$ & $\mathbf{1 0}$ & $\mathbf{1 1}$ & $\mathbf{1 2}$ \\
\hline \multirow{2}{*}{ Optimal solution (CPLEX) } & Objective & 63343 & 67683 & 70399 & 75133 & 78480 & 83410 & 86845 \\
& CPU time & 4.02 & 24.38 & 79.3 & 254.75 & 4526.35 & 92314 & 4000084 \\
\cline { 3 - 8 } & & & & & & & & \\
& Objective & 63343 & 67683 & 70399 & 75133 & 78480 & 83410 & 86845 \\
& CPU time & 2.25 & 9.58 & 43.23 & 127.3 & 1234 & 5796.75 & 84276 \\
Optimality gap & & & & & & & & \\
Improvement in CPU Time & & 0 & 0 & 0 & 0 & 0 & 0 & - \\
\hline
\end{tabular}

function and CPU time of UBRP is also shown in Table 1. As shown in Table 1, in all discussed problems, when five upper-bound rules are added to the set of constraints, the objective function of the problem is equal to the optimal solution, which is obtained by the CPLEX solver. Thus, there is no optimality gap between the solutions. This shows that no area of optimal solution space is removed by these rules. On the other hand, the results show that the CPU time is considerably reduced. This reduction in CPU time is the result of reduction in the number of branchings and also increasing the speed at which UBRP closes the opened nodes in the Branch and Bound tree. For more explanation on Branch and Bound, see [52]. These are the benefits of the proposed rules which are described as follows:

I. Reduction in the number of branchings on the nodes. In order to clarify, consider a node that the CPLEX solver branches on to create the two new sub-problems. The branching is on the value of variable $x_{t, t^{\prime}, s}$ whose value is fixed in the UBRP. Therefore, in UBRP, the branching on this variable does not occur. If the value of variable $x_{t, t^{\prime}, s}$ in CPLEX solver is integer, the node in the UBRP is also feasible, and compared to the CPLEX solver, the tree size from that node is halved. Also, the node is infeasible in UBRP if the value of $x_{t, t^{\prime}, s}$ is not integer, and so the tree does not expand more. Thus, by fixing binary variables, the number of branching decreases, and as a result, the CPU time decreases.

II. Reduction of time required to fathom the opened nodes. Fixing the binary variables decreases the required time to fathom the opened nodes. After a node opens, regarding the rules, the node is infeasible or some of their binary variables are known. Thus, in UBRP, the nodes are fathomed in shorter time compared to CPLEX. This process increases the speed of Branch and Bound algorithm and more nodes can be investigated in less time.
For more investigation, three diagrams of "number of opened nodes versus duality gap", "CPU time versus duality gap", and "CPU time versus number of opened nodes" are shown in Figures 8, 9, and 10, respectively. The duality gap in these figures refers to the gap between the upper and lower bounds, which can be obtained at each step of Branch and Bound algorithm.

As shown in Figure 8, in all problems with optimality gap equal to zero, the number of opened nodes in UBRP is lower than the CPLEX solver (as benefit I). Also, as shown in Figure 9, the duality in UBRP converges to zero quicker than in CPLEX. This is because more nodes are opened and fathomed as benefit II in shorter time, as mentioned earlier. As mentioned in benefit II, fixing binary variables increases the speed at which the nodes are fathomed, i.e. a higher number of nodes are fathomed at equal time. For more investigations, we proposed a measurement criterion as $\xi=C P U$ time/Opened nodes that measures the speed at which the opened nodes close. This criterion measures the impact of the proposed rules on the node fathoming speed. The amount of $\xi$ for each problem is shown in Table 2.

As shown in Table 2, criterion $\xi$ is reduced for all problems. In all problems, in order to reach a given duality gap, UBRP opens fewer nodes in a shorter period of time (except the problem with 12 trains stopped in 400000 seconds, and results of CPLEX are not known). The reason for this is as described in benefit I, showing that the proposed rules reduce the number of branching.

In order to clarify the improvement, consider the problem with nine trains. In this problem, in order to reach the duality gap about $0.5 \%$, CPLEX opened 305000 nodes in 206 seconds; however, the UBRP opened 160000 nodes (48\% of opened nodes in CPLEX) in 107 seconds (equal to $51 \%$ of CPLEX CPU time). From another view point, in the problem with eleven trains, UBRP opened 3057644 nodes after about 3775 seconds of solving process and the gap is about $0.99 \%$ 


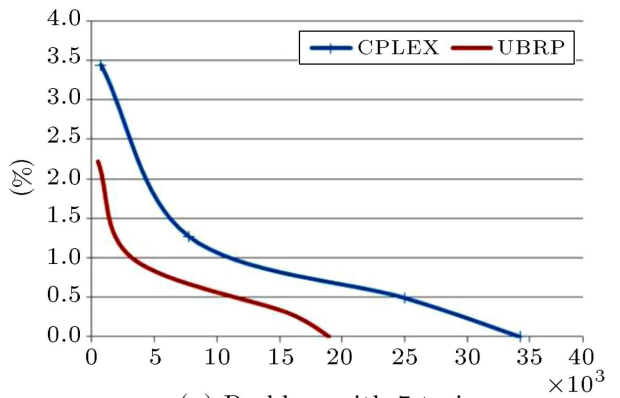

(a) Problem with 7 trains

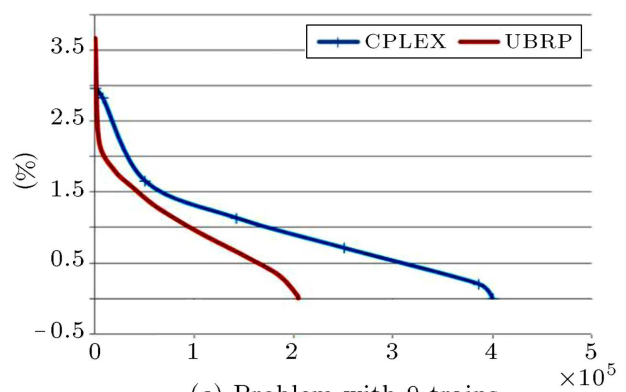

(c) Problem with 9 trains

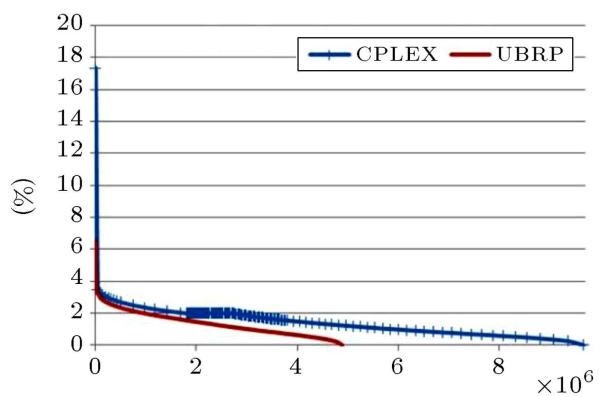

(e) Problem with 11 trains

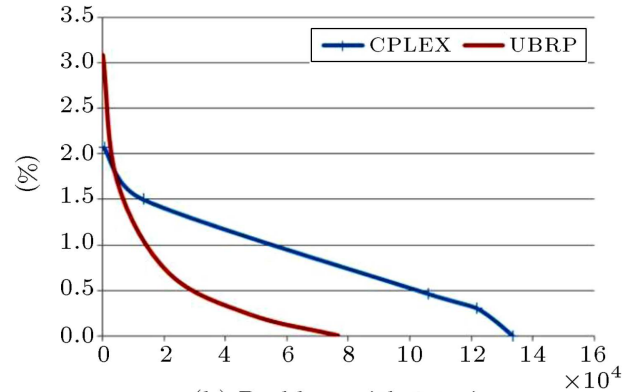

(b) Problem with 8 trains

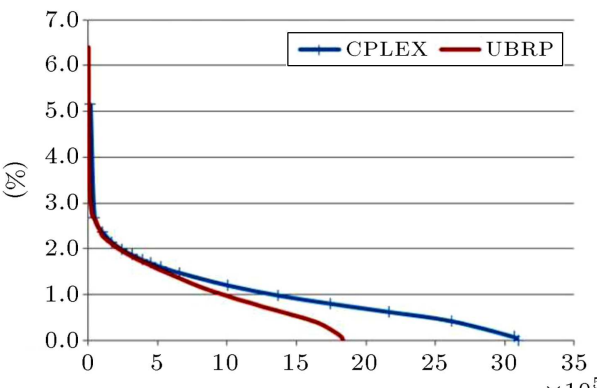

(d) Problem with 10 trains $\times 10^{5}$

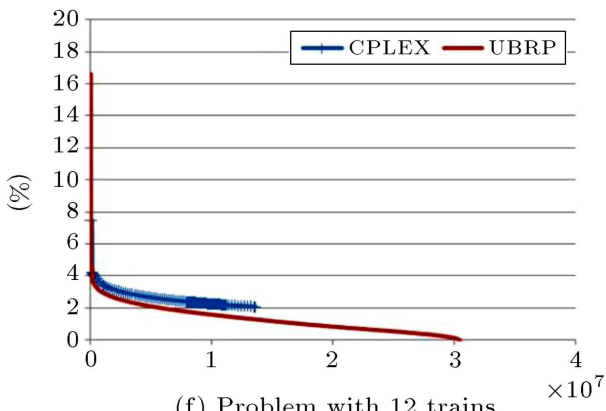

(f) Problem with 12 trains

Figure 8. Number of opened nodes versus duality gap for the optimal solution and UBRP.

Table 2. The amount of $\xi$ and number of binary variables for each problem.

\begin{tabular}{|c|c|c|c|c|c|c|c|c|}
\hline & \multicolumn{7}{|c|}{ Number of trains } \\
\hline & & 6 & 7 & 8 & 9 & 10 & 11 & 12 \\
\hline \multirow{4}{*}{ CPLEX } & CPU time (second) & 4.02 & 24.38 & 79.3 & 254.75 & 4526.4 & 92314 & 400084 \\
\hline & Number of opened nodes & 13864 & 34226 & 133384 & 400422 & 3097165 & 9672652 & 13630809 \\
\hline & CPU time/ opened nodes $(\xi)$ & 0.0019 & 0.0015 & 0.0016 & 0.0166 & 0.0481 & 0.0095 & 0.0294 \\
\hline & Number of discrete variables & 1212 & 1659 & 2176 & 2763 & 3420 & 4147 & 4944 \\
\hline \multirow{4}{*}{ UBRP } & CPU time (second) & 2.25 & 9.58 & 43.23 & 127.3 & 1234 & 5796.75 & 84276.3 \\
\hline & Number of opened nodes & 6356 & 18956 & 76532 & 204396 & 1835564 & 4890725 & 30505371 \\
\hline & CPU time/ opened nodes $(\xi)$ & 0.0004 & 0.0005 & 0.0006 & 0.0006 & 0.0007 & 0.0012 & 0.0028 \\
\hline & Number of discrete variables & 1138 & 1552 & 2044 & 2568 & 3181 & 3833 & 4568 \\
\hline $\begin{array}{c}\text { Improvement } \\
\text { percentage in } \xi\end{array}$ & & $82 \%$ & $67 \%$ & $64 \%$ & $96 \%$ & $99 \%$ & $88 \%$ & $91 \%$ \\
\hline
\end{tabular}

versus 6008691 opened nodes (is equal $51 \%$ of UBRP) in 52923 seconds (is equal $7.1 \%$, i.e., 1 to 14 of CPLEX) and $0.98 \%$ gap in CPLEX.

On the other hand, in an equal period of time, UBRP opens more nodes because of improvement in $\xi$. Figure 11 shows "CPU time versus number of opened nodes". As shown, in all problems except the problem with eight trains, in an equal period of time, UBRP opens more nodes and creates a smaller duality gap in that period of time. For example, in the problem with ten trains, after about 1290 seconds of solving process, UBRP opened 719791 nodes and the gap is 


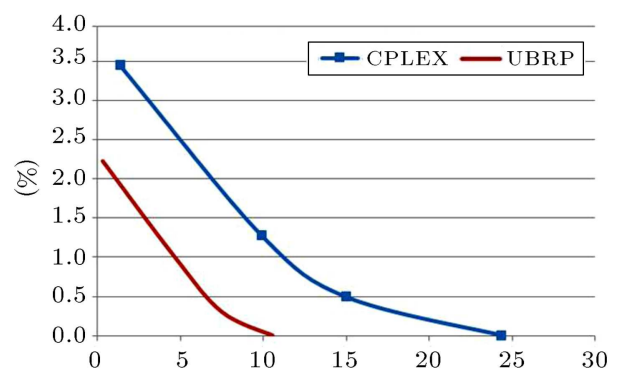

(a) Problem with 7 trains

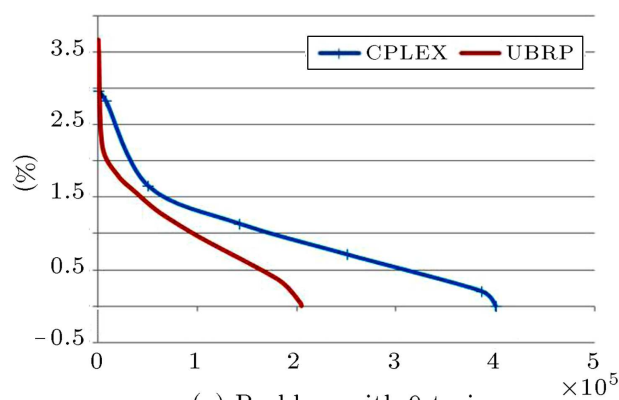

(c) Problem with 9 trains

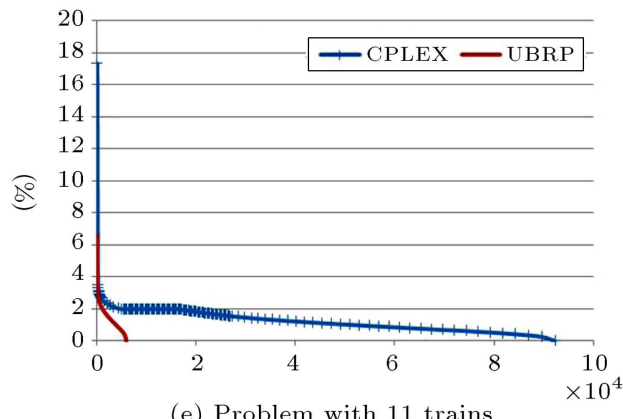

(e) Problem with 11 trains

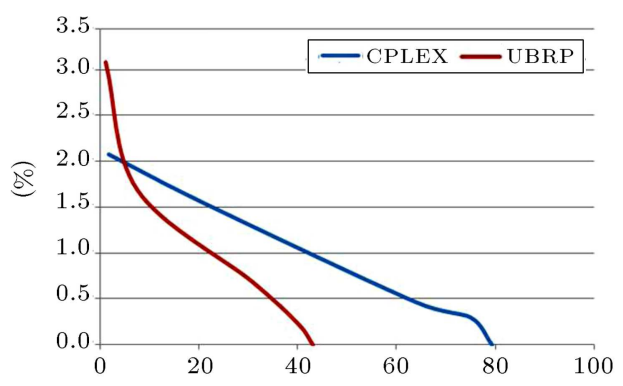

(b) Problem with 8 trains

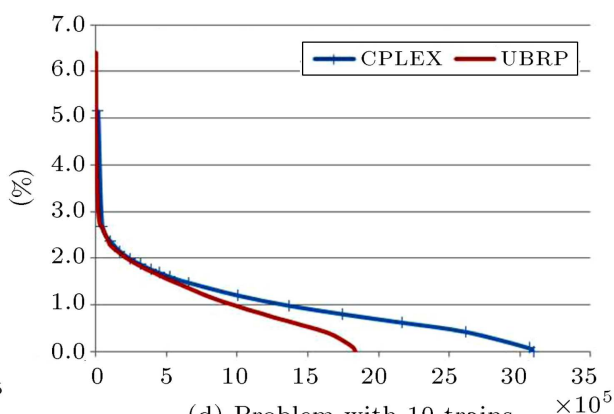

(d) Problem with 10 trains

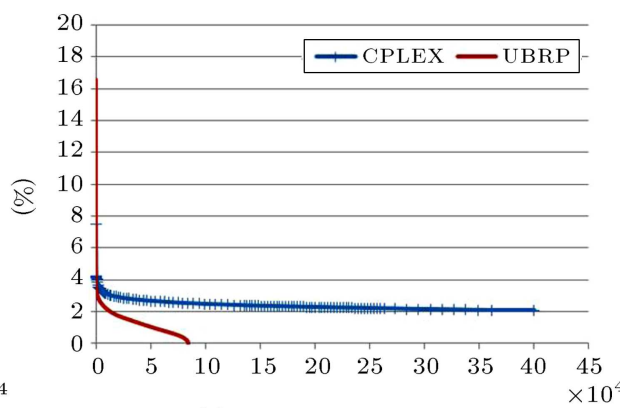

(f) Problem with 12 trains

Figure 9. CPU time versus duality gap in the optimal solution and UBRP.

about $0.74 \%$ versus 151211 opened nodes (equal to $21 \%$ of UBRP) and $1.13 \%$ gap in CPLEX (1.52 times greater than UBRP).

In sum, we can conclude that UBRP opens more nodes in an equal period of time while achieving a better duality gap and also the required number of the opened nodes to obtain the optimal solution is lower than CPLEX.

Last but not least, the number of discrete variables in CPLEX and UBRP is shown Figure 11.

Figure 11 also shows the trend of discrete variables in UBRP and CPLEX. It is obvious that the two trends go ahead, while the difference between the numbers of discrete variables increases. In other words, by increasing the number of trains, the number of relaxed discrete variables increases and that engenders the reduction of CPU time. Regarding the figure, the improvements in $\mathrm{CPU}$ time can be explained more clearly.

\subsection{Lower bound results}

In order to investigate the results of Lagrangian Relax- ation algorithm for a lower-bound, we used the same problem, which is described in the last section. The measurement criteria are the duality gap among the optimal value, the lower bound, and the CPU time. In order to obtain $U B$, a feasible solution is obtained as the mentioned procedure. Our algorithm starts with the result of the Relaxed Mixed Integer Programing (RMIP) model as a base for LR comparison and tries to improve it. Maximum number of the iterations in our investigation is set to 25 . In our investigations, there is only a little amount of improvement after iteration 15 and the algorithm tries to prove the optimality of the solution.

Results of LR model in Table 3 show that it has achieved the optimal value of problem and their values are the same as those reported by CPLEX in Table 1. The achievement stipulates the idea that the relaxed constraint has been correctly selected.

To investigate further the quality of the algorithm, the trend of the lower bound in iterations of the LR model for problems on 6 and 7 trains is shown in Figure 12. 


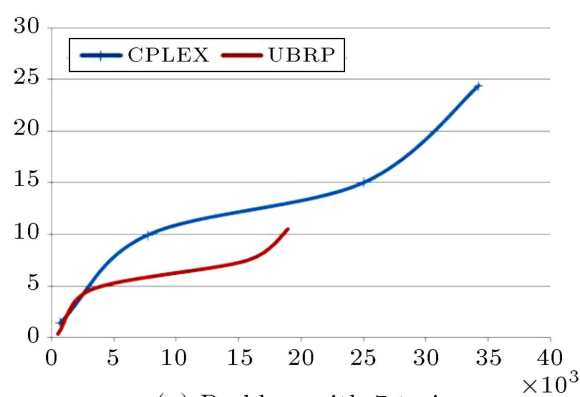

(a) Problem with 7 trains

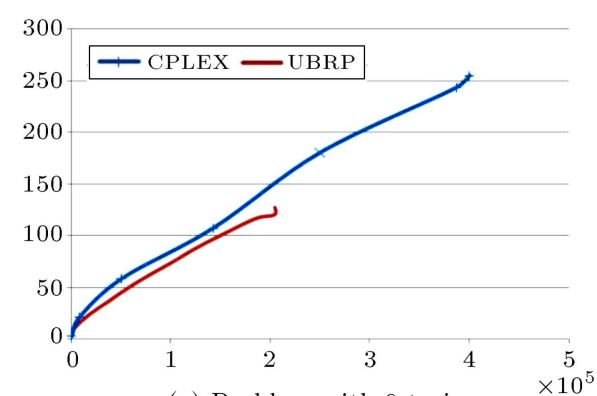

(c) Problem with 9 trains

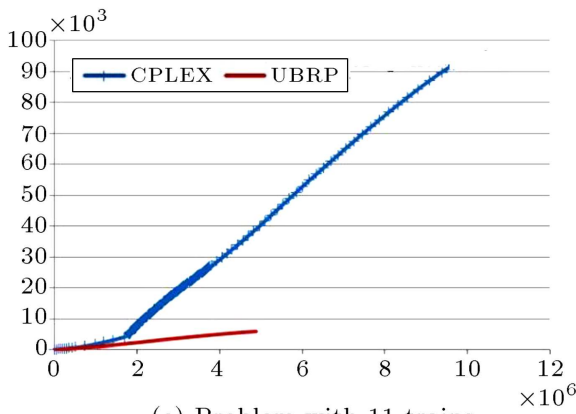

(e) Problem with 11 trains

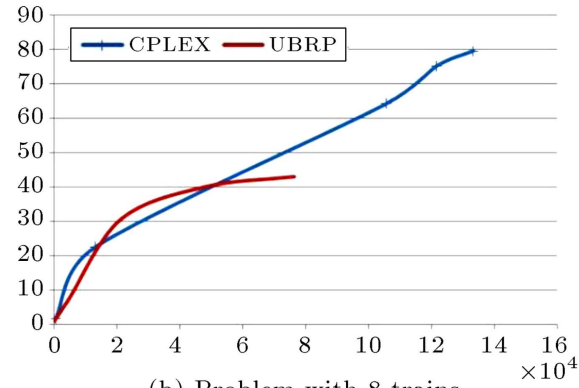

(b) Problem with 8 trains

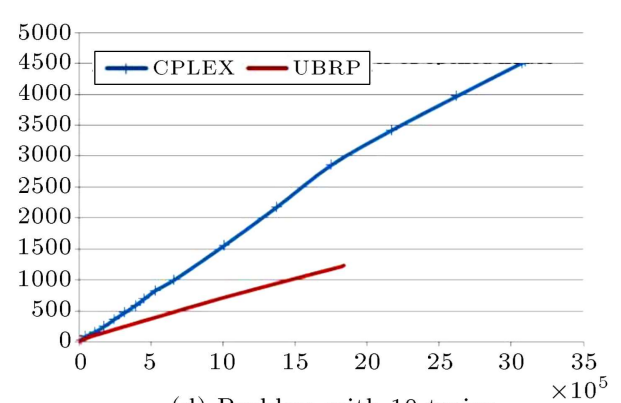

(d) Problem with 10 trains

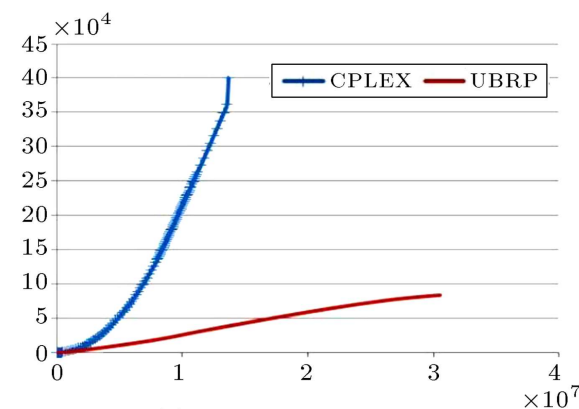

(f) Problem with 12 trains

Figure 10. CPU time versus number of opened nodes in the optimal solution and UBRP.

Table 3. Results of LR model.

\begin{tabular}{ccccc}
\hline & \multicolumn{4}{c}{ Number of trains } \\
\cline { 3 - 5 } & $\mathbf{5}$ & $\mathbf{6}$ & $\mathbf{7}$ \\
\hline \multirow{2}{*}{ LR model bound } & 59078 & 63343 & 67683 \\
& Number of iteration & 6 & 7 & 22 \\
& CPU time & $>3.35$ & $>4.02$ & $>24.38$ \\
\hline
\end{tabular}

Considering the fact that the selected relaxed constraint is in the form of equation and binds all the defined events together, it seems to be a very powerful constraint. As Figure 12 shows, the LR algorithm iteratively improves the lower bound until it gets the optimal solution. In addition, because the relaxed constraint is in an equality form, there is no fluctuation in the sign of $\gamma_{l, t, t^{\prime}, s}$, which results in a straightforward trend of improvement without fluctuation.

Nonetheless, the CPU time as another quality measurement criterion has not improved. In order to improve the CPU time, we defined another model using Constraint (16), which is a Special Ordered Set (SOS)

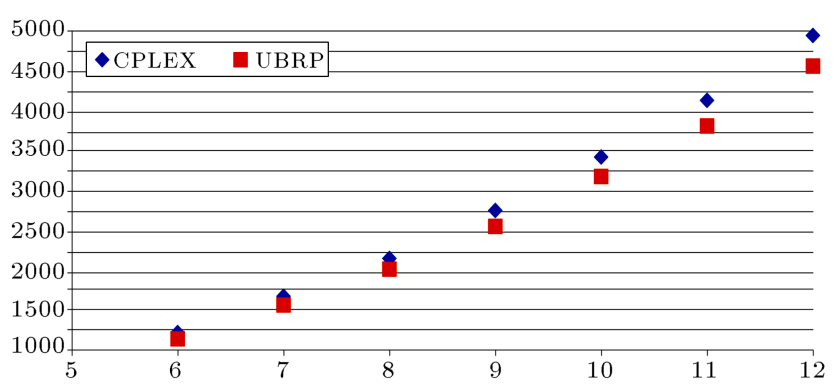

Figure 11. Number of discrete variables versus number of trains for CPLEX and UBRP.

of constraints and it considers the platforms capacity. The same experimental analysis was performed on that model. The result showed a fluctuation without any improving trend. Also, as another model, we relaxed Constraints (2) and (3). The idea was from the problem of Seoul metropolitan Railway network that [37] relaxed the constraint which connects the stations to each other. The result of their analysis was hopeful and encouraging; nevertheless, the model of the connecting constraints relaxed (Constraints (2) and (3)) does not result in an admirable solution in our 


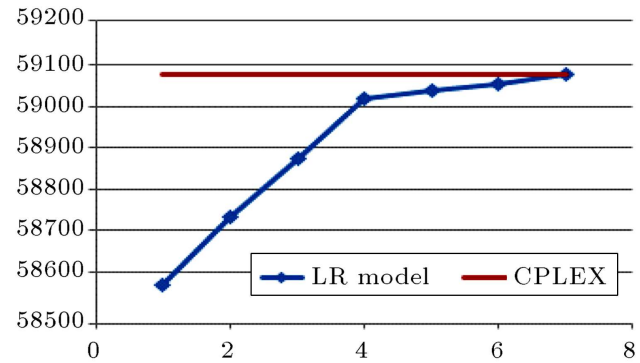

(a)

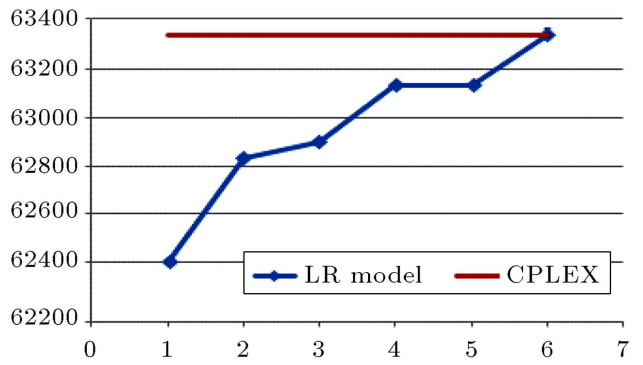

(b)

Figure 12. Trend of the Lagrangian relaxation lower bound: (a) For 5 trains; and (b) for 6 trains.

case. The result again showed a fluctuation without any improved trend.

\section{Conclusion}

In this study, a new MIP model for the railway scheduling problem is proposed that utilizes events of the railway networks. The general specifications and restrictions of railway networks are considered and also the station capacity constraints are embedded in the timetabling problem simultaneously. The model is NP-hard and is reduced to a flow-shop problem with block, machine assignment restriction, and start-time restrictions. Therefore, solving large-scale problems in an acceptable amount of time is not possible.

In order to reduce CPU time, some upper-bound rules based on the analysis of model's parameters have been presented. These rules consider the relation among the parameters of the model and try to eliminate some events which is not rationally wise to do. The results have added five constraints to the master problem, and experimental results testify that CPU time has been reduced up to $94 \%$ and the optimal solution also has been achieved. Furthermore, the analysis of the opened nodes shows that the rules can fathom more nodes in shorter time, which is indicative of its efficiency. These rules can be used on any other models by mapping the related variables.

By relaxing the constraint which ensures that only one of the events occurs each time, a Lagrangian Relaxation algorithm is proposed. In order to update the Lagrangian multipliers, we used the step-size method. Numerical analysis revealed the accuracy and efficiency of the proposed algorithm in a way that the optimal solution was achieved in all of the samples after about 20 iterations. Although the solving time of LR model is higher than the main problem, it induces some hope to improve the processing time by focusing more on the proposed Lagrangian Relaxation algorithm.

Our on-going research has focused on heuristic methods in order to find upper bounds. With regard to the new definition of decision variables, further research can be done on implementation of a decomposition method as Dantzig-Wolfe algorithm, benders decomposition or branch and price. Also, integrating the proposed rule in this article by a pricing algorithm will be worthwhile to use them in a Bisection algorithm.

\section{Acknowledgment}

We would like to show our appreciation of all the inspiring and productive comments made by Tehran Metro Agency. Further, we thank Prof. N. Salmasi for all his advice and encouragement which led to the favorable formation of this article. In the end, we are grateful to the authors for providing us with all the related cited papers that motivated us to the every end of the project.

\section{References}

1. Mu, S. and Dessouky, M. "Scheduling freight trains traveling on complex networks", Transportation Research Part B: Methodological, 45(7), pp. 1103-1123 (2011).

2. Higgins, A., Kozan, E. and Ferreira, L. "Optimal scheduling of trains on a single line track", Transportation Research Part B: Methodological, 30(2), pp. 147161 (1996).

3. Kroon, L.G. and Peeters, L.W.P.A "Variable trip time model for cyclic railway timetabling", Transportation Science (C) 2003 INFORMS, 37(2), pp. 198-212 (2003).

4. Ghoseiri, K., Szidarovszky, F. and Asgharpour, M.J. "A multi-objective train scheduling model and solution", Transportation Research Part B: Methodological, 38, pp. 927-952 (2004).

5. Zhou, X. and Zhong, M. "Bi-criteria train scheduling for high-speed passenger railroad planning applications", European Journal of Operational Research, 167(3), pp. 752-771 (2005).

6. Ping, R., Nan, L., Liqun, G., Zhiling, L. and Yang, L. "Application of particle swarm optimization to the train scheduling for high-speed passenger railroad planning", In IEEE International Symposium on Communications and Information Technology, 1, pp. 581584. (2005). 
7. Vansteenwegen, P. and Oudheusden, D.V. "Developing railway timetables which guarantee a better service", European Journal of Operational Research, 173(1), pp. 337-350 (2006).

8. Vansteenwegen, P. and Van Oudheusden, D. "Decreasing the passenger waiting time for an intercity rail network", Transportation Research Part B: Methodological, 41(4), pp. 478-492 (2007).

9. Li, X., Wang, D., Li, K. and Gao, Z. "A green train scheduling model and fuzzy multi-objective optimization algorithm", Applied Mathematical Modelling, 37(4), pp. 2063-2073 (2013).

10. Zhou, X. and Zhong, M. "Single-track train timetabling with guaranteed optimality: branchand-bound algorithms with enhanced lower bounds", Transportation Research Part B: Methodological, 41(3), pp. 320-341 (2007).

11. Lee, Y. and Chen, C.Y. "A heuristic for the train pathing and timetabling problem", Transportation Research Part B: Methodological, 43(8-9), pp. 837-851 (2009).

12. Castillo, E., Gallego, I., Urena, J.M. and Coronado, J.M. "Timetabling optimization of a single railway track line with sensitivity analysis", TOP, 17(2), pp. 256-287 (2009).

13. Yang, L., Gao, Z. and Li, K. "Passenger train scheduling on a single-track or partially double-track railway with stochastic information", Engineering Optimization, 42(11), pp. 1003-1022 (2010).

14. Castillo, E., Gallego, I., Urena, J. M. and Coronado, J.M. "Timetabling optimization of a mixed double and single-tracked railway network", Applied Mathematical Modelling, 35, pp. 859-878 (2011).

15. Castillo, E., Gallego, I., Sanchez-Cambronero, S., Menendez, J.M., Rivas, A., Nogal, M. and Grande, Z. "An alternate double-single track proposal for highspeed peripheral railway lines", Computer-Aided Civil and Infrastructure Engineering, 30(3), pp. 181-201 (2015).

16. Liu, S. Q. and Kozan, E. "Scheduling trains as a blocking parallel-machine job shop scheduling problem", Computers and Operations Research, 36(10), pp. 2840-2852 (2009).

17. Burdett, R.L. and Kozan, E. "Scheduling trains on parallel lines with crossover points", Journal of Intelligent Transportation Systems, 13(4), pp. 171-187 (2009a).

18. Burdett, R.L. and Kozan, E. "Techniques for restricting multiple overtaking conflicts and performing compound moves when constructing new train schedules", Mathematical and Computer Modelling, 50(1-2), pp. 314-328 (2009b).

19. Burdett, R.L. and Kozan, E. "A disjunctive graph model and framework for constructing new train schedules", European Journal of Operational Research, 200, pp. $85-98$ (2010a).
20. Burdett, R.L. and Kozan, E. "A sequencing approach for creating new train timetables", Springer-Verlag, OR Spectrum, 32, pp. 163-193 (2010b).

21. Caimi, G., Chudak, F., Fuchsberger, M., Laumanns, M. and Zenklusen, R. "A new resource-constrained multicommodity flow model for conflict-free train routing and scheduling", Transportation Science, 45(2), pp. 212-227 (2011).

22. Liu, S.Q. and Kozan, E. "Scheduling trains with priorities: A no-wait blocking parallel-machine job-shop scheduling model", Transportation Science, 45(2), pp. 175-198 (2011).

23. Furini, F. and Kidd, M.P. "A fast heuristic approach for train timetabling in a railway node", Electronic Notes in Discrete Mathematics, 41(0), pp. 205-212 (2013).

24. Tormos, P., Lova, A., Barber, F., Ingolotti, L., Abril, M. and Salido, M.A. "A genetic algorithm for railway scheduling problems", Studies in Computational Intelligence, 128, pp. 255-276 (2008).

25. Jamili, A., Shafia, M.A., Sadjadi, S.J. and TavakkoliMoghaddam, R. "Solving a periodic single-track train timetabling problem by an efficient hybrid algorithm", Engineering Applications of Artificial Intelligence, 25(4), pp. 793-800 (2012).

26. Shafia, M.A., Sadjadi, S.J., Jamili, A., TavakkoliMoghaddam, R. and Pourseyed-Aghaee, M. "The periodicity and robustness in a single-track train scheduling problem", Applied Soft Computing, 12(1), pp. 440452 (2012).

27. Cacchiani, V. and Toth, P. "Nominal and robust train timetabling problems", European Journal of Operational Research, 219(3), pp. 727-737 (2012).

28. Goerigk, M. "Exact and heuristic approaches to the robust periodic event scheduling problem", Public Transport, pp. 1-19 (2014).

29. Yang, L., Li, K., Gao, Z. and Li, X. "Optimizing trains movement on a railway network", Omega, 40(5), pp. 619-633 (2012).

30. Reimann, M. and Leal, J. "Single line train scheduling with ACO", In M. Middendorf and C. Blum (Eds.), Evolutionary Computation in Combinatorial Optimization, 7832, pp. 226-237: Springer Berlin Heidelberg (2013).

31. Xu, X., Li, K., Yang, L. and Ye, J. "Balanced train timetabling on a single-line railway with optimized velocity", Applied Mathematical Modelling, 38(3), pp. 894-909 (2014).

32. Sun, Y., Cao, C. and Wu, C. "Multi-objective optimization of train routing problem combined with train scheduling on a high-speed railway network", Transportation Research Part C: Emerging Technologies, 44(0), pp. 1-20 (2014).

33. Huang, Y., Yang, L., Tang, T., Cao, F. and Gao, Z. "Saving energy and improving service quality: bicriteria train scheduling in urban rail transit systems", IEEE Transactions on Intelligent Transportation Systems (2016). 
34. Heydar, M., Petering, M.E.H. and Bergmann, D.R. "Mixed integer programming for minimizing the period of a cyclic railway timetable for a single track with two train types", Computers and Industrial Engineering, 66(1), pp. 171-185 (2013).

35. Barrena, E., Canca, D., Coelho, L.C. and Laporte, G. "Exact formulations and algorithm for the train timetabling problem with dynamic demand", Computers and Operations Research, 44(0), pp. 66-74 (2014).

36. Khan, M.B. and Zhou, X. "Stochastic optimization model and solution algorithm for robust doubletrack train-timetabling problem", IEEE transactions on intelligent transportation systems, 11(1), pp. 81-89 (2010).

37. Min, Y.-H., Park, M.-J., Hong, S.-P. and Hong, S.-H. "An appraisal of a column-generation-based algorithm for centralized train-conflict resolution on a metropolitan railway network", Transportation Research Part B, 45, pp. 409-429 (2011).

38. Li, F., Gao, Z., Li, K. and Yang, L. "Efficient scheduling of railway traffic based on global information of train", Transportation Research Part B: Methodological, 42(10), pp. 1008-1030 (2008).

39. Dessouky, M.M., Lu, Q., Zhao, J. and Leachman, R.C. "An exact solution procedure to determine the optimal dispatching times for complex rail networks", IIE Transactions, 38(2), pp. 141-152 (2006).

40. Yang, L., Zhou, X. and Gao, Z. "Credibility-based rescheduling model in a double-track railway network: a fuzzy reliable optimization approach", Omega, 48, pp. 75-93 (2014).

41. Qi, J., Yang, L., Gao, Y., Li, S. and Gao, Z. "Integrated multi-track station layout design and train scheduling models on railway corridors", Transportation Research Part C: Emerging Technologies, 69, pp. 91-119 (2016).

42. Acuna-Agost, R., Michelon, P., Feillet, D. and Gueye, S. "A MIP-based local search method for the railway rescheduling problem", Networks, 57(1), pp. 69-86 (2011).

43. Tornquist, J. "Railway traffic disturbance management", Transportation Research Part A: Policy Pract, 41(3), pp. 249-266 (2007).

44. Tornquist, J. and Persson, A. "N-tracked railway traffic re-scheduling during disturbances", Transportation Research Part B: Methodological, 41(3), pp. 342-362 (2007).

45. Billionnet, A. "Using integer programming to solve the train-platforming problem", Transportation Science, 37(2), pp. 213-222 (2003).

46. Caimi, G., Burkolter, D. and Herrmann, T. "Finding delay-tolerant train routings through stations", In H.
Fleuren, D. den Hertog and P. Kort (Eds.), Operations Research Proceedings 2004, 2004, pp. 136-143: Springer Berlin Heidelberg (2005).

47. Carey, M. and Crawford, I. "Scheduling trains on a network of busy complex stations", Transportation Research Part B: Methodological, 41(2), pp. 159-178 (2007).

48. Chakroborty, P. and Vikram, D. "Optimum assignment of trains to platforms under partial schedule compliance", Transportation Research Part B: Methodological, 42(2), pp. 169-184 (2008).

49. Kroon, L.G., Edwin Romeijn, H. and Zwaneveld, P.J. "Routing trains through railway stations: complexity issues", European Journal of Operational Research, 98(3), pp. 485-498 (1997).

50. Carey, M. and Carville, S. "Scheduling and platforming trains at busy complex stations", Transportation Research Part A: Policy and Practice, 37(3), pp. 195224 (2003).

51. Zwaneveld, P.J., Kroon, L.G. and van Hoesel, S. P. M. "Routing trains through a railway station based on a node packing model", European Journal of Operational Research, 128(1), pp. 14-33 (2001).

52. Pinedo, M.L., Scheduling: Theory, Algorithms, and Systems", (3rd Ed.), Springer (2008).

53. Garey, M.R., Johnson, D.S. and Sethi, R. "The complexity of flowshop and jobshop scheduling", Mathematics of Operations Research, 1(2), pp. 117-129 (1976).

54. Guan, X.H., Zhai, Q.Z. and Lai, F. "New Lagrangian relaxation based algorithm for resource scheduling with homogeneous subproblems", Journal of Optimization Theory and Applications, 113(1), pp. 65-82 (2002).

55. Brännlund, U., Lindberg, P.O., Nõu, A. and Nilsson, J.-E. "Railway timetabling using Lagrangian relaxation", Transportation Science, 32(4), pp. 358-369 (1998).

56. Chen, H. and Luh, P.B. "An alternative framework to Lagrangian relaxation approach for job shop scheduling", European Journal of Operational Research, 149(3), pp. 499-512 (2003).

57. Tang, L., Xuan, H. and Liu, J. "A new Lagrangian relaxation algorithm for hybrid flowshop scheduling to minimize total weighted completion time", Computers and Operations Research, 33(11), pp. 3344-3359 (2006).

\section{Biographies}

Afshin Oroojlooyjadid is a PhD Student of Industrial Engineering at the Department of Industrial Engineering in Lehigh University, Bethlehem, PA, USA. He received his MSc degree from the Sharif University of Technology in the Industrial Engineering and his BSc degree in the same field from Isfahan University of 
Technology. His main areas of research interests include reinforcement learning, supply chain management, and mixed integer programming.

Kourosh Eshghi is a Professor of Industrial Engineering Department at Sharif University of Technology,
Tehran, Iran. He received his $\mathrm{PhD}$ degree in the field of Operations Research from University of Toronto in 1997. He was the Head of IE Department for 7 years. His field of interests includes graph theory, integer programming, and facility location. He is the author of 4 books and over 80 international journal papers. 